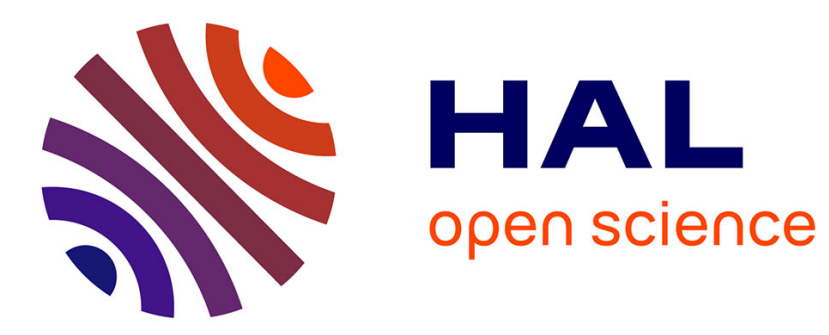

\title{
Well-posedness and energy decay for Timoshenko systems with discrete time delay under frictional damping and/or infinite memory in the displacement
}

Aissa Guesmia

\section{- To cite this version:}

Aissa Guesmia. Well-posedness and energy decay for Timoshenko systems with discrete time delay under frictional damping and/or infinite memory in the displacement. Afrika Matematika, 2017, 28 (7), pp.1253-1284. 10.1007/s13370-017-0514-8 . hal-02891549

\section{HAL Id: hal-02891549 \\ https://hal.science/hal-02891549}

Submitted on 9 Aug 2020

HAL is a multi-disciplinary open access archive for the deposit and dissemination of scientific research documents, whether they are published or not. The documents may come from teaching and research institutions in France or abroad, or from public or private research centers.
L'archive ouverte pluridisciplinaire HAL, est destinée au dépôt et à la diffusion de documents scientifiques de niveau recherche, publiés ou non, émanant des établissements d'enseignement et de recherche français ou étrangers, des laboratoires publics ou privés. 
Dear Author,

Here are the proofs of your article.

- You can submit your corrections online, via e-mail or by fax.

- For online submission please insert your corrections in the online correction form. Always indicate the line number to which the correction refers.

- You can also insert your corrections in the proof PDF and email the annotated PDF.

- For fax submission, please ensure that your corrections are clearly legible. Use a fine black pen and write the correction in the margin, not too close to the edge of the page.

- Remember to note the journal title, article number, and your name when sending your response via e-mail or fax.

- Check the metadata sheet to make sure that the header information, especially author names and the corresponding affiliations are correctly shown.

- Check the questions that may have arisen during copy editing and insert your answers/ corrections.

- Check that the text is complete and that all figures, tables and their legends are included. Also check the accuracy of special characters, equations, and electronic supplementary material if applicable. If necessary refer to the Edited manuscript.

- The publication of inaccurate data such as dosages and units can have serious consequences. Please take particular care that all such details are correct.

- Please do not make changes that involve only matters of style. We have generally introduced forms that follow the journal's style.

Substantial changes in content, e.g., new results, corrected values, title and authorship are not allowed without the approval of the responsible editor. In such a case, please contact the Editorial Office and return his/her consent together with the proof.

- If we do not receive your corrections within $\mathbf{4 8}$ hours, we will send you a reminder.

- Your article will be published Online First approximately one week after receipt of your corrected proofs. This is the official first publication citable with the DOI. Further changes are, therefore, not possible.

- The printed version will follow in a forthcoming issue.

\section{Please note}

After online publication, subscribers (personal/institutional) to this journal will have access to the complete article via the DOI using the URL: http://dx.doi.org/[DOI].

If you would like to know when your article has been published online, take advantage of our free alert service. For registration and further information go to: http://www.link.springer.com.

Due to the electronic nature of the procedure, the manuscript and the original figures will only be returned to you on special request. When you return your corrections, please inform us if you would like to have these documents returned. 


\section{Metadata of the article that will be visualized in OnlineFirst}

\begin{tabular}{|c|c|}
\hline ArticleTitle & $\begin{array}{l}\text { Well-posedness and energy decay for Timoshenko systems with discrete time delay under frictional } \\
\text { damping and/or infinite memory in the displacement }\end{array}$ \\
\hline \multicolumn{2}{|l|}{ Article Sub-Title } \\
\hline Article CopyRight & $\begin{array}{l}\text { African Mathematical Union and Springer-Verlag GmbH Deutschland } \\
\text { (This will be the copyright line in the final PDF) }\end{array}$ \\
\hline Journal Name & Afrika Matematika \\
\hline \multirow[t]{12}{*}{ Corresponding Author } & Family Name \\
\hline & Particle \\
\hline & Given Name \\
\hline & Suffix \\
\hline & Institut Elie Cartan de Lorraine (IECL), UMR 7502 \\
\hline & Université de Lorraine \\
\hline & Bat. A, Ile du Saulcy, 57045, Metz Cedex 01, France \\
\hline & Phone \\
\hline & Fax \\
\hline & aissa.guesmia@univ-lorraine.fr \\
\hline & URL \\
\hline & ORCID \\
\hline & 24 April 2017 \\
\hline \multirow[t]{2}{*}{ Schedule } & Revised \\
\hline & 23 June 2017 \\
\hline Abstract & $\begin{array}{l}\text { In this paper, we consider a vibrating system of Timoshenko-type in a bounded one-dimensional domain } \\
\text { with discrete time delay and complementary frictional damping and infinite memory controls all acting on } \\
\text { the transversal displacement. We show that the system is well-posed in the sens of semigroup and that, } \\
\text { under appropriate assumptions on the weights of the delay and the history data, the stability of the system } \\
\text { holds in case of the equal-speed propagation as well as in the opposite case in spite of the presence of a } \\
\text { discrete time delay, where the decay rate of solutions is given in terms of the smoothness of the initial data } \\
\text { and the growth of the relaxation kernel at infinity. The results of this paper extend the ones obtained by the } \\
\text { present author and Messaoudi in (Acta Math Sci } 36: 1-33,2016 \text { ) to the case of presence of discrete delay. }\end{array}$ \\
\hline Keywords (separated by '-') & $\begin{array}{l}\text { Well-posedness - General decay - Time delay - Infinite memory - Frictional damping - Viscoelastic - } \\
\text { Timoshenko-type - Semigroup theory - Energy method }\end{array}$ \\
\hline $\begin{array}{l}\text { Mathematics Subject } \\
\text { Classification (separated by } \\
\text { '-') }\end{array}$ & 35B37 - 35L55 - 74D05 - 93D15 - 93D20 \\
\hline Footnote Information & \\
\hline
\end{tabular}




\title{
Well-posedness and energy decay for Timoshenko systems with discrete time delay under frictional damping and/or infinite memory in the displacement
}

\author{
Aissa Guesmia ${ }^{1}$
}

Received: 24 April 2017 / Accepted: 23 June 2017

(C) African Mathematical Union and Springer-Verlag GmbH Deutschland 2017

\begin{abstract}
In this paper, we consider a vibrating system of Timoshenko-type in a bounded one-dimensional domain with discrete time delay and complementary frictional damping and infinite memory controls all acting on the transversal displacement. We show that the system is well-posed in the sens of semigroup and that, under appropriate assumptions on the weights of the delay and the history data, the stability of the system holds in case of the equal-speed propagation as well as in the opposite case in spite of the presence of a discrete time delay, where the decay rate of solutions is given in terms of the smoothness of the initial data and the growth of the relaxation kernel at infinity. The results of this paper extend the ones obtained by the present author and Messaoudi in (Acta Math Sci 36:1-33, 2016) to the case of presence of discrete delay.
\end{abstract}

Keywords Well-posedness · General decay · Time delay · Infinite memory · Frictional damping $\cdot$ Viscoelastic $\cdot$ Timoshenko-type $\cdot$ Semigroup theory $\cdot$ Energy method

Mathematics Subject Classification 35B37 - 35L55 - 74D05 - 93D15 - 93D20

\section{Introduction}

In this paper, we are concerned with the well-posedness and the long-time behavior of the solution of the following Timoshenko system:

Aissa Guesmia

aissa.guesmia@univ-lorraine.fr

1 Institut Elie Cartan de Lorraine (IECL), UMR 7502, Université de Lorraine, Bat. A, Ile du Saulcy, 57045 Metz Cedex 01, France 


$$
\left\{\begin{array}{l}
\rho_{1} \varphi_{t t}(x, t)-k_{1}\left(\varphi_{x}(x, t)+\psi(x, t)\right)_{x}+d(x) \varphi_{t}(x, t-\tau)+b(x) \varphi_{t}(x, t) \\
\quad+\int_{0}^{+\infty} g(s)\left(a(x) \varphi_{x}(x, t-s)\right)_{x} d s=0, \\
\rho_{2} \psi_{t t}(x, t)-k_{2} \psi_{x x}(x, t)+k_{1}\left(\varphi_{x}(x, t)+\psi(x, t)\right)=0, \\
\varphi(0, t)=\psi_{x}(0, t)=\varphi(L, t)=\psi_{x}(L, t)=0, \\
\varphi(x,-t)=\varphi_{0}(x, t), \varphi_{t}(x, 0)=\varphi_{1}(x), \varphi_{t}(x,-\tau p)=f_{0}(x,-\tau p), \\
\psi(x, 0)=\psi_{0}(x), \psi_{t}(x, 0)=\psi_{1}(x),
\end{array}\right.
$$

for $(x, t, p) \in] 0, L[\times] 0,+\infty[\times] 0,1\left[, d:[0, L] \rightarrow \mathbb{R}, a, b:[0, L] \rightarrow \mathbb{R}_{+}\right.$and $g: \mathbb{R}_{+} \rightarrow \mathbb{R}_{+}$are given functions (to be specified later), where $\mathbb{R}_{+}=\left[0,+\infty\left[, L, \tau, \rho_{i}, k_{i}\right.\right.$ $(i=1,2)$ are positive constants,

$$
\left.\varphi_{0}:\right] 0, L[\times]-\infty, 0\left[\rightarrow \mathbb{R}, \quad \varphi_{1}, \psi_{0}, \psi_{1}:\right] 0, L\left[\rightarrow \mathbb{R} \quad \text { and } \quad f_{0}:\right] 0, L[\times]-\tau, 0[\rightarrow \mathbb{R}
$$

are given initial data, and

$$
(\varphi, \psi):] 0, L[\times] 0,+\infty\left[\rightarrow \mathbb{R}^{2}\right.
$$

is the state of (1.1). A subscript $y$ and the notation $\partial_{y}$ denote the derivative with respect to $y$. We also use the prime notation to denote the derivative when the function has only one variable. The infinite integral in (1.1), $b(x) \varphi_{t}(x, t)$ and $d(x) \varphi_{t}(x, t-\tau)$ represent, respectively, the infinite memory, the frictional damping and the discrete time delay. For simplicity of notation, the space and time variables are used only when it is necessary to avoid ambiguity.

Our aim is the study of the well-posedness and asymptotic behavior of the solutions of (1.1) in case of the equal-speed propagation

$$
\frac{k_{1}}{\rho_{1}}=\frac{k_{2}}{\rho_{2}}
$$

as well as in the opposite case. The equality (1.2) means that the first two equations in (1.1) have the same speeds of wave propagation $\sqrt{\frac{k_{1}}{\rho_{1}}}$ and $\sqrt{\frac{k_{2}}{\rho_{2}}}$, respectively.

Timoshenko [69], in 1921, introduced the following model to describe the transverse vibration of a beam:

$$
\begin{cases}\rho u_{t t}=\left(K\left(u_{x}-\varphi\right)\right)_{x}, & \text { in }] 0, L[\times] 0,+\infty[ \\ I_{\rho} \varphi_{t t}=\left(E I \varphi_{x}\right)_{x}+K\left(u_{x}-\varphi\right), & \text { in }] 0, L[\times] 0,+\infty[\end{cases}
$$

where $t$ denotes the time variable and $x$ is the space variable along the beam of length $L$, in its equilibrium configuration, $u$ is the transverse displacement of the beam and $\varphi$ is the rotation angle of the filament of the beam. The coefficients $\rho, I_{\rho}, E, I$ and $K$ are, respectively, the density (the mass per unit length), the polar moment of inertia of a cross section, Young's modulus of elasticity, the moment of inertia of a cross section, and the shear modulus. Since then, this model has attracted the attention of many researchers and an important amount of work has been devoted to the issue of the stabilization and the search for the minimum dissipation by which the solutions decay uniformly to the stable state as time goes to infinity. To achieve this goal, diverse types of dissipative mechanisms have been used and several stability results have been obtained. We mention some of these results (for more results, we refer the reader to the list of references of this paper, which is not exhaustive, and the references therein).

Absence of delay: $d \equiv 0$. In the case of presence of controls on both the rotation angle and the transverse displacement, investigations showed that the Timoshenko systems are stable without any restriction on the constants $\rho_{1}, \rho_{2}, k_{1}$ and $k_{2}$. In this regards, many decay 
estimates were obtained; see $[26,31,39,40,56]$. However, in the case of only one control on the rotation angle, the rate of decay depends heavily on the constants $\rho_{1}, \rho_{2}, k_{1}$ and $k_{2}$ and the regularity of the initial data. Precisely, if (1.2) holds, the results obtained are similar to those established for the case of the presence of controls in both equations. We quote in this regard $[2,7,14,21-24,26,41,42,45-47,63]$. But, if (1.2) does not hold, a situation which is more interesting from the physics point of view, then it has been shown that the Timoshenko system is not exponentially stable even for exponentially decaying relaxation functions or linear frictional damping, and only weak decay estimates can be obtained for regular solutions in the presence of dissipation. This has been demonstrated in [2,14,23,24,26,43], for the case of finite or infinite memory, and in [17,22], for complementary frictional damping and finite or infinite memory acting on the rotation angle equation. We also refer the reader to [55] (and its references) concerning the stability of Timoshenko-type systems in $\mathbb{R}$ (instaed of $] 0, L[$ ) with controls acting on the rotation angle.

For the stability of Timoshenko systems via heat effect, we mention the pioneer work [44] devoted to the study of the following system:

$$
\begin{cases}\rho_{1} \varphi_{t t}-\sigma\left(\varphi_{x}, \psi\right)_{x}=0, & \text { in }] 0, L[\times] 0,+\infty[, \\ \rho_{2} \psi_{t t}-b \psi_{x x}+k\left(\varphi_{x}+\psi\right)+\gamma \theta_{x}=0, & \text { in }] 0, L[\times] 0,+\infty[, \\ \rho_{3} \theta_{t}-k \theta_{x x}+\gamma \psi_{t x}=0, & \text { in }] 0, L[\times] 0,+\infty[,\end{cases}
$$

where $\theta$ denotes the temperature difference. In their work, Rivera and Racke [44] established, under appropriate conditions on the function $\sigma$ and the positive constants $\rho_{i}, b, k$ and $\gamma$, several exponential decay results for the linearized system with various boundary conditions. They also proved a non-exponential stability result for the case of non-equal speed of propagation, and proved an exponential decay result for the nonlinear case. Guesmia et al. [27] discussed a linear version of (1.4) and completed the work of [44] by establishing some polynomial decay results in the case of non-equal speed of propagation.

In (1.4), the heat flux is given by Fourier's law. As a result, this theory predicts an infinite speed of heat propagation; that is, any thermal disturbance at one point has an instantaneous effect elsewhere in the body. Experiments showed that heat conduction in some dielectric crystals at low temperatures is free of this paradox and disturbances, which are almost entirely thermal, propagate in a finite speed. This phenomenon in dielectric crystals is called second sound. To overcome this physical paradox, many theories have merged. One of which suggests that we should replace Fourier's law by Cattaneo's law. In line with this theory, (1.4), in its linear form, becomes

$$
\begin{cases}\rho_{1} \varphi_{t t}-k_{1}\left(\varphi_{x}+\psi\right)_{x}=0, & \text { in }] 0, L[\times] 0,+\infty[, \\ \rho_{2} \psi_{t t}-k_{2} \psi_{x x}+k_{1}\left(\varphi_{x}+\psi\right)+\delta \theta_{x}=0, & \text { in }] 0, L[\times] 0,+\infty[, \\ \rho_{3} \theta_{t}+\gamma q_{x}+\delta \psi_{t x}=0, & \text { in }] 0, L[\times] 0,+\infty[, \\ \tau q_{t}+q+k \theta_{x}=0, & \text { in }] 0, L[\times] 0,+\infty[,\end{cases}
$$

where $q$ denotes the heat flux. Fernández Sare and Racke [15] studied (1.5) and proved that (1.2) is no longer sufficient to obtain exponential stability even in the presence of an extra viscoelastic dissipation of the form $\int_{0}^{+\infty} g(s) \psi_{x x}(t-s) d s$ in the second equation. Very recently, Santos et al. [62] considered (1.5), introduced a new stability number

$$
\chi=\left(\tau-\frac{\rho_{1}}{k_{1} \rho_{3}}\right)\left(\rho_{2}-\frac{k_{2} \rho_{1}}{k_{1}}\right)-\frac{\tau \rho_{1} \delta^{2}}{k_{1} \rho_{3}}
$$


and used the semigroup method to obtain an exponential decay result, for $\chi=0$, and a polynomial decay, for $\chi \neq 0$. See, also [26,29,30,39,54,58,59]. Notice that, when $\tau=0$ (Fourier's law), $\chi=0$ if and only if (1.2) holds.

In all above mentioned works, the stability was either via both equation control or the angular rotation equation control. Recently, Almeida Júnior et al. [4] considered the situation when the control is only on the transverse displacement equation, which is more realistic from the physics point of view. Precisely, they looked into the following system:

$$
\begin{cases}\rho_{1} \varphi_{t t}-k_{1}\left(\varphi_{x}+\psi\right)_{x}+\mu \varphi_{t}=0, & \text { in }] 0, L[\times] 0,+\infty[ \\ \rho_{2} \psi_{t t}-k_{2} \psi_{x x}+k_{1}\left(\varphi_{x}+\psi\right)=0, & \text { in }] 0, L[\times] 0,+\infty[\end{cases}
$$

where $\mu$ is a positive constant, and showed that the linear frictional damping $\mu \varphi_{t}$ is strong enough to obtain exponential stability of (1.7) provided that (1.2) holds. They, also, proved some non-exponential and polynomial decay results in the case of non-equal speed situation. The results of [4] were, very recently, extended in [25] to the case where the linear frictional damping $\mu \varphi_{t}$ is replaced by a nonlinear one and/or an infinite memory. The same authors of [4] considered in [5]

$$
\begin{cases}\rho_{1} \varphi_{t t}-\kappa\left(\varphi_{x}+\psi\right)_{x}+\sigma \theta_{x}=0, & \text { in }] 0, L[\times] 0,+\infty[, \\ \rho_{2} \psi_{t t}-b \psi_{x x}+\kappa\left(\varphi_{x}+\psi\right)-\sigma \theta=0, & \text { in }] 0, L[\times] 0,+\infty[, \\ \rho_{3} \theta_{t}-\gamma \theta_{x x}+\sigma\left(\varphi_{x}+\psi\right)_{t}=0, & \text { in }] 0, L[\times] 0,+\infty[,\end{cases}
$$

with various boundary conditions, and established the exponential stability of (1.8) for equal-speed case, and non-exponential stability for the opposite case. In the case of lack of exponential stability, they proved some algebraic (polynomial) stability for strong solutions.

Presence of delay: $d \neq 0$. The questions related to well-posedness and stability/instability of Timoshenko-type systems as well as evolution equations with time delay have attracted considerable attention in recent years and many researchers have shown that the time delay can destabilize a system that was asymptotically stable in the absence of time delay.

When the delay and controls are present on the rotation angle equation, we mention the following Timoshenko system:

$$
\left\{\begin{array}{l}
\rho_{1} \varphi_{t t}-k_{1}\left(\varphi_{x}+\psi\right)_{x}=0, \\
\rho_{2} \psi_{t t}-k_{2} \psi_{x x}+k_{1}\left(\varphi_{x}+\psi\right)+\int_{0}^{t} g(s) \psi_{x x}(t-s) d s+\mu_{1} \psi_{t}+\mu_{2} \psi_{t}(t-\tau)=0,
\end{array}\right.
$$

in ]0, $1[\times] 0,+\infty\left[\right.$, studied in [57], where $\mu_{1}, \mu_{2}$ and $\tau$ are fixed non-negative constants. The author of [57] proved the stability of (1.9) under the assumptions (1.2) and $0<\mu_{2} \leq \mu_{1}$, where the decay rate of solutions depends on the one of $g$. The obtained stability results in [57] generalize the ones of [60] concerning (1.9) in the case $g \equiv 0$ and $0<\mu_{2}<\mu_{1}$, and they were generalized in [32] to the case $g \equiv 0$ and variable time delay $\tau(t)$. In [61], the stability of Timoshenko systems with two internal time delays and two boundary linear feedbacks was proved under some smallness conditions on $L$ and the weights of the delays.

When no frictional damping is present, the stability of this Timoshenko system

$$
\left\{\begin{array}{l}
\rho_{1} \varphi_{t t}-k_{1}\left(\varphi_{x}+\psi\right)_{x}=0 \\
\rho_{2} \psi_{t t}-k_{2} \psi_{x x}+k_{1}\left(\varphi_{x}+\psi\right)+\int_{0}^{+\infty} g(s) \psi_{x x}(t-s) d s+D(\psi)=0,
\end{array}\right.
$$


in ]0, $L[\times] 0,+\infty[$, was proved in [20], in both discrete time delay case

$$
D(\psi)=\mu_{2} \psi_{t}(t-\tau)
$$

and distributed one

$$
D(\psi)=\int_{0}^{+\infty} f(s) \psi_{t}(t-s) d s,
$$

where $\mu_{2} \in \mathbb{R}^{*}$ and $f: \mathbb{R}_{+} \rightarrow \mathbb{R}$ is a given function. In contrast to the situation of absence of delay and/or presence of frictional damping, (1.10) is not necessarily dissipative with respect to its classical energy. To overcome subsequently the difficulties generated by the non-dissipativeness character of (1.10), some new functionals were introduced in [20] to get crucial estimates on some terms generated by the time delay and the infinite memory. The results of [20] generalizes the ones of [18] concerning the particular case $D(\psi)=\mu_{2} \psi_{t}(t-\tau)$ and $g$ converges exponentially to zero at infinity.

Similar stability results for various hyperbolic evolution equations with frictional damping and/or memory and/or time delay exist in the literature, in this regard, we refer the reader to $[1,3,6,8-10,12,13,16,19,28,34-38,48-52,64-68]$.

As far as we know, the problem of stability of Timoshenko system with a time delay under infinite memory and/or frictional damping all acting on the transversal displacement has never been treated in the literature. Our goal in this paper is to investigate the effect of each control on the asymptotic behavior of the solutions of (1.1) in the presence of a time delay, and on the decay rate of its energy, when both controls are acting cooperatively, allowing each control to vanish on the whole domain. To our best knowledge, this situation has never been considered before in the literature. Under appropriate assumptions on the history data $\varphi_{0}$, we give an explicit characterization of the decay rate of solutions depending on the growth of $g$ at infinity and the following relations between the weights $b$ and $d$ of, respectively, the frictional damping and time delay:

$$
\inf _{[0, L]}(b-|d|)>0
$$

and

$$
\inf _{[0, L]}(b-|d|) \leq 0 .
$$

Contrarly to the case (1.11), system (1.1) is not necessarily dissipative with respect to its classical energy when (1.12) holds (see (4.1) and (4.2) below). This creates some difficulties and, so, we prove the exponential stability of (1.1) provided that (1.2) holds, $g$ converges exponetially to zero at infinity and $\|d\|_{\infty}$ is small enough. In the case when (1.11) holds, we give two general decay estimates (corresponding to the case (1.2) and the opposite one) depending on the smoothness of initial data and growth of $g$ at infinity characterized by the condition (2.9) below introduced in [16]. These results give a generalization of the ones proved by the present author and Messaoudi in [25] concerning the case $d \equiv 0$.

The proof of the well-posedness is based on the maximal monotone operators and semigroup approach (see, for example [33,53]). However, the proof of stability estimates is based on the multiplier method combined with some integral or differential inequalities (see, for example [1,3,10,33-37]) and an approach introduced in [16,19], for a class of abstract hyperbolic systems of single or coupled equations with one infinite memory. In the case when (1.2) does not hold, we use also some ideas given in $[3,14,17]$ to get the decay rate of solutions in terms of the regularity of initial data and the general growth of $g$ at infinity. 
The paper is organized as follows. In Sect. 2, we set up the hypotheses and present our wellposedness and stability results. In Sect. 3, we prove the well-posedness result. In Sect. 4, we establish some lemmas needed for the proof of the stability results which will be completed in Sect. 5 when (1.2) and (1.11) hold, in Sect. 6 when (1.2) and (1.12) hold, and in Sect. 7 when (1.2) does not hold and (1.11) holds. Finally, some general comments and issues will be given in Sect. 8 .

\section{Preliminaries and obtained results}

\subsection{Hypotheses}

We consider the following hypotheses:

(H1) The functions $a, b:[0, L] \rightarrow \mathbb{R}_{+}$and $d:[0, L] \rightarrow \mathbb{R}$ are such that

$$
\begin{aligned}
& a \in C^{1}([0, L]), b, d \in C([0, L]), \\
& \inf _{[0, L]}(a+b)>0, \\
& a \equiv 0 \text { or } \inf _{[0, L]} a>0 .
\end{aligned}
$$

(H2) The function $g: \mathbb{R}_{+} \rightarrow \mathbb{R}_{+}$is a non-increasing of class $C^{1}\left(\mathbb{R}_{+}\right)$such that $g(0)>0$ and

$$
g_{0}\|a\|_{\infty}<\frac{k_{1} k_{2}}{k_{0} k_{1}+k_{2}}
$$

where $g_{0}=\int_{0}^{+\infty} g(s) d s$ and $k_{0}$ is the smallest constant depending only on $L$ and satisfying (Poincaré's inequality)

$$
\int_{0}^{L} v^{2} d x \leq k_{0} \int_{0}^{L} v_{x}^{2} d x, \quad \forall v \in H_{*}^{1}(] 0, L[)
$$

with

$$
H_{*}^{1}(] 0, L[)=\left\{v \in H^{1}(] 0, L[), \int_{0}^{L} v d x=0\right\} .
$$

(H3) There exist a positive constant $\alpha$ and an increasing strictly convex function $G$ : $\mathbb{R}_{+} \rightarrow \mathbb{R}_{+}$of class $C^{1}\left(\mathbb{R}_{+}\right) \cap C^{2}(] 0,+\infty[)$ satisfying

$$
G(0)=G^{\prime}(0)=0 \text { and } \lim _{t \rightarrow+\infty} G^{\prime}(t)=+\infty
$$

such that

$$
g^{\prime}(t) \leq-\alpha g(t), \quad \forall t \geq 0
$$

or

$$
\int_{0}^{+\infty} \frac{g(t)}{G^{-1}\left(-g^{\prime}(t)\right)} d t+\sup _{t \in \mathbb{R}_{+}} \frac{g(t)}{G^{-1}\left(-g^{\prime}(t)\right)}<+\infty .
$$

Remark 2.1 1. The hypothesis (2.9) was introduced in [16] and it allows a wider class of relaxation functions than the ones considered in $[14,43]$ (see examples given in $[16,26]$ ). 
2. As in [25], using the second equation and boundary conditions in (1.1), we easily verify that

$$
\partial_{t t}\left(\int_{0}^{L} \psi d x\right)+\frac{k_{1}}{\rho_{2}} \int_{0}^{L} \psi d x=0 .
$$

By solving this ordinary differential equation and using the initial data of $\psi$, we find

$$
\int_{0}^{L} \psi d x=\left(\int_{0}^{L} \psi_{0} d x\right) \cos \left(\sqrt{\frac{k_{1}}{\rho_{2}}} t\right)+\sqrt{\frac{\rho_{2}}{k_{1}}}\left(\int_{0}^{L} \psi_{1} d x\right) \sin \left(\sqrt{\frac{k_{1}}{\rho_{2}}} t\right) .
$$

Let

$$
\tilde{\psi}=\psi-\frac{1}{L}\left(\int_{0}^{L} \psi_{0} d x\right) \cos \left(\sqrt{\frac{k_{1}}{\rho_{2}}} t\right)-\frac{1}{L} \sqrt{\frac{\rho_{2}}{k_{1}}}\left(\int_{0}^{L} \psi_{1} d x\right) \sin \left(\sqrt{\frac{k_{1}}{\rho_{2}}} t\right) .
$$

Then, one can easily check that

$$
\int_{0}^{L} \tilde{\psi} d x=0
$$

and, hence, Poincaré's inequality (2.5) is applicable for $\tilde{\psi}$ provided that $\tilde{\psi} \in H^{1}(] 0, L[)$. In addition, $(\varphi, \tilde{\psi})$ satisfies $(1.1)$ with initial data

$$
\tilde{\psi}_{0}=\psi_{0}-\frac{1}{L} \int_{0}^{L} \psi_{0} d x \text { and } \tilde{\psi}_{1}=\psi_{1}-\frac{1}{L} \int_{0}^{L} \psi_{1} d x
$$

instead of $\psi_{0}$ and $\psi_{1}$, respectively. In the sequel, we work with $\tilde{\psi}$ instead of $\psi$, but, for simplicity of notation, we use $\psi$ instead of $\tilde{\psi}$.

3. Thanks to Poincaré's inequality (2.5) (applied for $\psi \in H_{*}^{1}(] 0, L[)$ ), we have

$$
k_{1} \int_{0}^{L}\left(\varphi_{x}+\psi\right)^{2} d x \geq k_{1}(1-\hat{\epsilon}) \int_{0}^{L} \varphi_{x}^{2} d x+k_{0} k_{1}\left(1-\frac{1}{\hat{\epsilon}}\right) \int_{0}^{L} \psi_{x}^{2} d x,
$$

for any $0<\hat{\epsilon}<1$. Then, according to (2.4), we can choose $\hat{\epsilon}>0$ such that

$$
\frac{k_{0} k_{1}}{k_{0} k_{1}+k_{2}}<\hat{\epsilon}<\frac{1}{k_{1}}\left(k_{1}-g_{0}\|a\|_{\infty}\right)
$$

and deduce from (2.13) that

$$
\hat{k} \int_{0}^{L}\left(\varphi_{x}^{2}+\psi_{x}^{2}\right) d x \leq \int_{0}^{L}\left(-g_{0}\|a\|_{\infty} \varphi_{x}^{2}+k_{2} \psi_{x}^{2}+k_{1}\left(\varphi_{x}+\psi\right)^{2}\right) d x,
$$

where $\hat{k}=\min \left\{k_{1}(1-\hat{\epsilon})-g_{0}\|a\|_{\infty}, k_{2}+k_{0} k_{1}\left(1-\frac{1}{\hat{\epsilon}}\right)\right\}>0$.

Because $\int_{0}^{L} \varphi_{x}^{2} d x$ and $\int_{0}^{L} \psi_{x}^{2} d x$ define norms, for $\varphi$ and $\psi$ on $H_{0}^{1}(] 0, L[)$ and $H_{*}^{1}(] 0, L[)$, respectively, then

$$
\int_{0}^{L}\left(-g_{0}\|a\|_{\infty} \varphi_{x}^{2}+k_{2} \psi_{x}^{2}+k_{1}\left(\varphi_{x}+\psi\right)^{2}\right) d x
$$

defines a norm on $H_{0}^{1}(] 0, L[) \times H_{*}^{1}(] 0, L[)$, for $(\varphi, \psi)$, equivalent to the one induced by $\left(H^{1}(] 0, L[)\right)^{2}$. 


\subsection{Well-posedness}

We give here a brief idea about the formulation of (1.1) into an abstract first order system and the related existence, uniqueness and smoothness of solution. Following the ideas of $[11,48]$, let

$$
\eta(x, t, s)=\varphi(x, t)-\varphi(x, t-s), \quad \text { for } \quad(x, t, s) \in] 0, L[\times] 0,+\infty[\times] 0,+\infty[
$$

and

$$
\left.z(x, t, p)=\varphi_{t}(x, t-\tau p), \quad \text { for } \quad(x, t, p) \in\right] 0, L[\times] 0,+\infty[\times] 0,1[\text {. }
$$

Then

$$
\begin{aligned}
& \begin{cases}\eta_{t}+\eta_{s}-\varphi_{t}=0, & \text { in }] 0, L[\times] 0,+\infty[\times] 0,+\infty[, \\
\eta(0, t, s)=\eta(L, t, s)=0, & \text { in }] 0,+\infty[\times] 0,+\infty[, \\
\eta(x, t, 0)=0, & \text { in }] 0, L[\times] 0,+\infty[,\end{cases} \\
& \begin{cases}\tau z_{t}+z_{p}=0, & \text { in }] 0, L[\times] 0,+\infty[\times] 0,1[, \\
z(x, t, 0)=\varphi_{t}(x, t), & \text { in }] 0, L[\times] 0,+\infty[, \\
z(x, t, 1)=\varphi_{t}(x, t-\tau), & \text { in }] 0, L[\times] 0,+\infty[\end{cases}
\end{aligned}
$$

and

$$
\begin{cases}\eta_{0}(x, s):=\eta(x, 0, s)=\varphi_{0}(x, 0)-\varphi_{0}(x, s), & \text { in }] 0, L[\times] 0,+\infty[, \\ z_{0}(x, p):=z(x, 0, p)=f_{0}(x,-\tau p), & \text { in }] 0, L[\times] 0,1[.\end{cases}
$$

Let

$$
\begin{aligned}
& U=\left(\varphi, \psi, \varphi_{t}, \psi_{t}, z, \eta\right)^{T}, \\
& U_{0}=\left(\varphi_{0}(\cdot, 0), \psi_{0}, \varphi_{1}, \psi_{1}, z_{0}, \eta_{0}\right)^{T}
\end{aligned}
$$

and

$$
\mathcal{H}=H_{0}^{1}(] 0, L[) \times H_{*}^{1}(] 0, L[) \times L^{2}(] 0, L[) \times L_{*}^{2}(] 0, L[) \times L_{\xi} \times L_{g},
$$

where

$$
\begin{aligned}
L_{*}^{2}(] 0, L[) & =\left\{v \in L^{2}(] 0, L[), \int_{0}^{L} v d x=0\right\}, \\
L_{g} & =\left\{v: \mathbb{R}_{+} \rightarrow H_{0}^{1}(] 0, L[), \int_{0}^{L} a \int_{0}^{+\infty} g(s) v_{x}^{2}(s) d s d x<+\infty\right\}, \\
L_{\xi} & =\{v:] 0,1\left[\rightarrow L^{2}(] 0, L[), \int_{0}^{L} \xi \int_{0}^{1} v^{2}(p) d p d x<+\infty\right\}
\end{aligned}
$$

and $\xi:[0, L] \rightarrow \mathbb{R}_{+}$defined by

$$
\xi= \begin{cases}\tau b & \text { if (1.11) holds and } d \neq 0 \\ \tau\|d\|_{\infty} & \text { if (1.12) holds or } d \equiv 0\end{cases}
$$

The spaces $L_{g}$ and $L_{\xi}$ endowed with the inner products

$$
\langle v, w\rangle_{L_{g}}=\int_{0}^{L} a \int_{0}^{+\infty} g(s) v_{x}(s) w_{x}(s) d s d x
$$

\section{Springer}


and

$$
\langle v, w\rangle_{L_{\xi}}=\int_{0}^{L} \xi \int_{0}^{1} v(p) w(p) d p d x
$$

are Hilbert spaces by vertue of the following Poincaré's inequality:

$$
\exists \tilde{k}_{0}>0: \quad \int_{0}^{L} v^{2} d x \leq \tilde{k}_{0} \int_{0}^{L} v_{x}^{2} d x, \quad \forall v \in H_{0}^{1}(] 0, L[)
$$

and the fact that $a>0$ if $a \neq 0$ (according to (2.3)), and $\xi>0$ if $d \neq 0$ (by vertue of (2.25)). The space $\mathcal{H}$ is equipped with the inner product defined by

$$
\begin{aligned}
\langle V, W\rangle_{\mathcal{H}}= & \left\langle v_{6}, w_{6}\right\rangle_{L_{g}}+\left\langle v_{5}, w_{5}\right\rangle_{L_{\xi}}+k_{1} \int_{0}^{L}\left(\partial_{x} v_{1}+v_{2}\right)\left(\partial_{x} w_{1}+w_{2}\right) d x \\
& +\int_{0}^{L}\left(-g_{0} a \partial_{x} v_{1} \partial_{x} w_{1}+k_{2} \partial_{x} v_{2} \partial_{x} w_{2}+\rho_{1} v_{3} w_{3}+\rho_{2} v_{4} w_{4}\right) d x,
\end{aligned}
$$

for any $V=\left(v_{1}, \ldots, v_{6}\right)^{T} \in \mathcal{H}$ and $W=\left(w_{1}, \ldots, w_{6}\right)^{T} \in \mathcal{H}$. Because $L_{g}$ and $L_{\xi}$ are Hilbert spaces, then also $\mathcal{H}$ is a Hilbert space according to (2.14).

Now, we define the linear operators $B$ and $A$ by

$$
B\left(v_{1}, \ldots, v_{6}\right)^{T}=-\frac{\xi_{0}}{\rho_{1}}\left(0,0, v_{3}, 0,0,0\right)^{T},
$$

where

$$
\xi_{0}= \begin{cases}0 & \text { if (1.11) holds } \\ \|d\|_{\infty} & \text { if (1.12) holds }\end{cases}
$$

and

$$
A\left(\begin{array}{c}
v_{1} \\
v_{2} \\
v_{3} \\
v_{4} \\
v_{5} \\
v_{6}
\end{array}\right)=\left(\begin{array}{c}
-v_{3} \\
-v_{4} \\
-\frac{k_{1}}{\rho_{1}} \partial_{x}\left(\partial_{x} v_{1}+v_{2}\right)+\frac{g_{0}}{\rho_{1}} \partial_{x}\left(a \partial_{x} v_{1}\right)+\frac{b+\xi_{0}}{\rho_{1}} v_{3}+\frac{d}{\rho_{1}} v_{5}(1)-\frac{1}{\rho_{1}} \int_{0}^{+\infty} g(s) \partial_{x}\left(a \partial_{x} v_{6}(s)\right) d s \\
-\frac{k_{2}}{\rho_{2}} \partial_{x x} v_{2}+\frac{k_{1}}{\rho_{2}}\left(\partial_{x} v_{1}+v_{2}\right) \\
\frac{1}{\tau} \partial_{p} v_{5} \\
-v_{3}+\partial_{s} v_{6}
\end{array}\right) .
$$

The system (1.1) is equivalent to

$$
\left\{\begin{array}{l}
\left.U^{\prime}(t)+(A+B) U(t)=0 \text { on }\right] 0,+\infty[, \\
U(0)=U_{0}
\end{array}\right.
$$

The domain of $B$ is given by $D(B)=\mathcal{H}$. However, the domain of $A$ is defined by $D(A)=\left\{V=\left(v_{1}, \ldots, v_{6}\right)^{T} \in \mathcal{H}, A V \in \mathcal{H}, \partial_{x} v_{2}(0)=\partial_{x} v_{2}(L)=0, v_{5}(0)=v_{3}, v_{6}(0)=0\right\}$ and it can be characterized by

$$
\begin{aligned}
& D(A)=\left\{\left(v_{1}, \ldots, v_{6}\right)^{T} \in H_{0}^{1}(] 0, L[) \times\left(H^{2}(] 0, L[) \cap H_{*}^{1}(] 0, L[)\right) \times H_{0}^{1}(] 0, L[)\right. \\
& \quad \times H_{*}^{1}(] 0, L[) \times L_{\xi} \times L_{g}, k_{1} \partial_{x x} v_{1}-g_{0} \partial_{x}\left(a \partial_{x} v_{1}\right)+\int_{0}^{+\infty} g(s) \partial_{x}\left(a \partial_{x} v_{6}(s)\right) d s \in L^{2}(] 0, L[), \\
& \left.\partial_{p} v_{5} \in L_{\xi}, \partial_{s} v_{6} \in L_{g}, \partial_{x} v_{2}(0)=\partial_{x} v_{2}(L)=0, v_{5}(0)=v_{3}, v_{6}(0)=0\right\} .
\end{aligned}
$$


We use the classical notation $D\left(A^{0}\right)=\mathcal{H}, D\left(A^{1}\right)=D(A)$ and

$$
D\left(A^{n}\right)=\left\{V \in D\left(A^{n-1}\right), A V \in D\left(A^{n-1}\right)\right\}, \quad \text { for } n=2,3, \ldots,
$$

endowed with the graph norm $\|V\|_{D\left(A^{n}\right)}=\sum_{k=0}^{n}\left\|A^{k} V\right\|_{\mathcal{H}}$.

Remark 2.2 If $a \equiv 0$ (resp. $d \equiv 0$ ), the variable $\eta$ (resp. $z$ ) is not considered, and therefore, the corresponding components in the definition of $U, U_{0}, \mathcal{H}, B, A$ and $D(A)$ will not appear.

Our well-posedness result reads as follows:

Theorem 2.3 Assume that (H1)-(H3) are satisfied. For any $n \in \mathbb{N}$ and $U_{0} \in D\left(A^{n}\right)$, the system (2.29) has a unique solution

$$
U \in \cap_{k=0}^{n} C^{n-k}\left(\mathbb{R}_{+} ; D\left(A^{k}\right)\right) .
$$

\subsection{Stability}

The energy functional associated with (1.1) is defined by

$$
\begin{aligned}
E(t):= & \frac{1}{2}\|U(t)\|_{\mathcal{H}}^{2} \\
= & \frac{1}{2}\left(g \circ \varphi_{x}\right)(t)+\frac{1}{2} \int_{0}^{L} \xi \int_{0}^{1} \varphi_{t}^{2}(t-\tau p) d p d x \\
& +\frac{1}{2} \int_{0}^{L}\left(\rho_{1} \varphi_{t}^{2}+\rho_{2} \psi_{t}^{2}+k_{1}\left(\varphi_{x}+\psi\right)^{2}+k_{2} \psi_{x}^{2}-g_{0} a \varphi_{x}^{2}\right) d x,
\end{aligned}
$$

where

$$
(\phi \circ v)(t)=\int_{0}^{L} a \int_{0}^{+\infty} \phi(s)(v(t)-v(t-s))^{2} d s d x,
$$

for any $v: \mathbb{R} \rightarrow L^{2}(] 0, L[)$ and $\phi: \mathbb{R}_{+} \rightarrow \mathbb{R}_{+}$.

Now, we give our first stability result which concerns the case when (1.2) and (1.11) hold.

Theorem 2.4 Assume that (1.2), (1.11) and (H1)-(H3) are satisfied and let $U_{0} \in \mathcal{H}$ such that

$$
\sup _{t \in \mathbb{R}_{+}} \int_{t}^{+\infty} \frac{g(s)}{G^{-1}\left(-g^{\prime}(s)\right)} \int_{0}^{L} \varphi_{0 x}^{2}(s-t) d x d s<+\infty .
$$

Then there exist positive constants $\epsilon_{0}, \alpha_{1}$ and $\alpha_{2}$, for which $E$ satisfies

$$
E(t) \leq \alpha_{1} \tilde{G}^{-1}\left(\alpha_{2} t\right), \quad \forall t \in \mathbb{R}_{+},
$$

where $\tilde{G}(t)=\int_{t}^{1} \frac{1}{G_{0}(s)} d s$ and

$$
G_{0}(s)= \begin{cases}s & \text { if (2.8) holds, } \\ s G^{\prime}\left(\epsilon_{0} s\right) & \text { if (2.9) holds. }\end{cases}
$$

Remark 2.5 1. Because $\lim _{t \rightarrow 0^{+}} \tilde{G}(t)=+\infty$ (by vertue of (H3)), then (2.34) implies that

$$
\lim _{t \rightarrow+\infty} E(t)=0 \text {. }
$$

\section{Springer}


2. In case (2.8), $\tilde{G}(s)=-\ln s$ and (2.34) is reduced to

$$
E(t) \leq \alpha_{1} e^{-\alpha_{2} t}, \quad \forall t \in \mathbb{R}_{+},
$$

which is the best decay rate given by (2.34). For specific examples of decay rates given by (2.34), see [17].

Our second stability result concerns the case when (1.2) and (1.12) hold.

Theorem 2.6 Assume that (1.2), (1.12), (H1) and (H2) are satisfied and

$$
\inf _{[0, L]} a>0 \text { and (2.8) holds. }
$$

Then there exists a positive constant $d_{0}$ independent of $d$ such that, if

$$
\|d\|_{\infty}^{2}+\|d\|_{\infty}<d_{0}
$$

then, for any $U_{0} \in \mathcal{H}$, there exist positive constants $\alpha_{1}$ and $\alpha_{2}$, for which $E$ satisfies (2.37).

When (1.2) does not hold and (1.11) holds, we prove the following stability result:

Theorem 2.7 Assume that (1.11) and (H1)-(H3) are satisfied. Let $n \in \mathbb{N}^{*}$ and $U_{0} \in D\left(A^{n}\right)$ such that

$$
\sup _{t \in \mathbb{R}_{+}} \max _{k=0, \ldots, n} \int_{t}^{+\infty} \frac{g(s)}{G^{-1}\left(-g^{\prime}(s)\right)} \int_{0}^{L}\left(\frac{\partial^{k} \varphi_{0 x}(s-t)}{\partial s^{k}}\right)^{2} d x d s<+\infty .
$$

Then there exist positive constant $\epsilon_{0}$ and $c_{n}$ such that $E$ satisfies

$$
E(t) \leq G_{n}\left(\frac{c_{n}}{t}\right), \quad \forall t>0,
$$

where $G_{m}(s)=G_{1}\left(s G_{m-1}(s)\right)$, for $m=2, \ldots, n$ and $s \in \mathbb{R}_{+}, G_{1}=G_{0}^{-1}$ and $G_{0}$ is defined in (2.35).

Remark 2.8 When (2.8) holds, $G_{n}(s)=s^{n}$ and (2.41) becomes

$$
E(t) \leq \frac{c_{n}}{t^{n}}, \quad \forall t>0
$$

which is the best decay rate given by (2.41). For specific examples of decay rates given by (2.41), see [19].

\section{Well-posedness}

The proof of Theorem 2.3 is based on the semigroup appraoch by proving that $A+B$ generates a $C_{0}$-semigroup in $\mathcal{H}$. We consider the case $\inf _{[0, L]} a>0$ and $d \neq 0$; the proof in cases $a \equiv 0$ and/or $d \equiv 0$ is similar and simpler.

First, we prove that $-A$ is dissipative. Let $V=\left(v_{1}, \ldots, v_{6}\right)^{T} \in D(A)$. Exploiting the definition of $D(A)$ and integrating by parts, we find

$$
\begin{aligned}
\langle-A V, V\rangle_{\mathcal{H}}= & -\frac{1}{2} \int_{0}^{L} a \int_{0}^{+\infty} g(s) \partial_{s}\left(\partial_{x} v_{6}(s)\right)^{2} d s d x-\frac{1}{2 \tau} \int_{0}^{L} \xi \int_{0}^{1} \partial_{p}\left(v_{5}(p)\right)^{2} d p d x \\
& -\int_{0}^{L}\left(b+\xi_{0}\right) v_{3}^{2} d x-\int_{0}^{L} d v_{3} v_{5}(1) d x .
\end{aligned}
$$


Integrating by parts for the first two terms of the above equality, using Young's inequality

$$
\lambda_{1} \lambda_{2} \leq \frac{\lambda}{2} \lambda_{1}^{2}+\frac{1}{2 \lambda} \lambda_{2}^{2}, \quad \forall \lambda_{1}, \lambda_{2} \in \mathbb{R}, \forall \lambda>0
$$

(with $\lambda_{1}=\left|v_{3}\right|, \lambda_{2}=\left|v_{5}(1)\right|$ and $\lambda=1$ ) and noting that $v_{5}(0)=v_{3}$ and $v_{6}(0)=0$ (from the definition of $D(A)$ ), we get

$$
\begin{aligned}
\langle-A V, V\rangle_{\mathcal{H}} \leq & \frac{1}{2} \int_{0}^{L} a \int_{0}^{+\infty} g^{\prime}(s)\left(\partial_{x} v_{6}(s)\right)^{2} d s d x \\
& +\int_{0}^{L}\left(-b-\xi_{0}+\frac{\xi}{2 \tau}+\frac{|d|}{2}\right) v_{3}^{2} d x+\int_{0}^{L}\left(\frac{|d|}{2}-\frac{\xi}{2 \tau}\right) v_{5}^{2}(1) d x .(3.2)
\end{aligned}
$$

The definitions (2.25) and (2.28) of $\xi$ and $\xi_{0}$ imply that, if (1.11) holds and $d \neq 0$,

$$
-b-\xi_{0}+\frac{\xi}{2 \tau}+\frac{|d|}{2}=\frac{|d|}{2}-\frac{\xi}{2 \tau}=\frac{|d|-b}{2} \leq 0,
$$

and, if (1.12) holds or $d \equiv 0$,

$$
-b-\xi_{0}+\frac{\xi}{2 \tau}+\frac{|d|}{2}=-b+\frac{|d|-\|d\|_{\infty}}{2} \leq 0 \text { and } \frac{|d|}{2}-\frac{\xi}{2 \tau}=\frac{|d|-\|d\|_{\infty}}{2} \leq 0 .
$$

Consequently, the last two integrals in (3.2) are non-positive. Therefore

$$
\langle-A V, V\rangle_{\mathcal{H}} \leq \frac{1}{2} \int_{0}^{L} a \int_{0}^{+\infty} g^{\prime}(s)\left(\partial_{x} v_{6}(s)\right)^{2} d s d x \leq 0,
$$

since $g$ is non-increasing. Then $-A$ is dissipative.

Second, we whow that $I d+A$ is surjective. For this purpose, let $F=\left(f_{1}, \ldots, f_{6}\right)^{T} \in \mathcal{H}$, we seek $V=\left(v_{1}, \ldots, v_{6}\right)^{T} \in D(A)$ satisfying

$$
(I d+A) V=F .
$$

The first two equations in (3.4) are equivalent to

$$
\left\{\begin{array}{l}
v_{3}=v_{1}-f_{1} \\
v_{4}=v_{2}-f_{2}
\end{array}\right.
$$

Using the first equation in (3.5), the last two equations in (3.4) are equivalent to

$$
\left\{\begin{array}{l}
v_{5}+\frac{1}{\tau} \partial_{p} v_{5}=f_{5}, \\
v_{6}+\partial_{s} v_{6}=v_{1}-f_{1}+f_{6}
\end{array}\right.
$$

then, by solving the ordinary differential equations (3.6) and noting that $v_{5}(0)=v_{3}=v_{1}-f_{1}$ and $v_{6}(0)=0($ see definition of $D(A))$, we get

$$
v_{5}=\left(v_{1}-f_{1}+\tau \int_{0}^{p} f_{5}(y) e^{\tau y} d y\right) e^{-\tau p}=e^{-\tau p} v_{1}-\left(f_{1}-\tau \int_{0}^{p} f_{5}(y) e^{\tau y} d y\right) e^{-\tau p}
$$

and

$$
v_{6}=\left(\int_{0}^{s} e^{y}\left(v_{1}-f_{1}+f_{6}(y)\right) d y\right) e^{-s}=\left(1-e^{-s}\right) v_{1}-\left(\int_{0}^{s} e^{y}\left(f_{1}-f_{6}(y)\right) d y\right) e^{-s} .
$$

\section{Springer}


We see that, if

$$
\left(v_{1}, v_{2}\right) \in H_{0}^{1}(] 0, L[) \times\left(H^{2}(] 0, L[) \cap H_{*}^{1}(] 0, L[)\right),
$$

then, from (3.5) to (3.8), we have $\left(v_{3}, v_{4}\right) \in H_{0}^{1}(] 0, L[) \times H_{*}^{1}(] 0, L[),\left(v_{5}, v_{6}\right) \in L_{\xi} \times L_{g}$, $\left(\partial_{p} v_{5}, \partial_{s} v_{6}\right) \in L_{\xi} \times L_{g}, v_{5}(0)=v_{3}$ and $v_{6}(0)=0$.

Next, plugging (3.5) and (3.7) into the third and fourth equations in (3.4), we get

$$
\left\{\begin{array}{l}
\frac{1}{\rho_{1}}\left(\rho_{1}+b+\xi_{0}+d e^{-\tau}\right) v_{1}-\frac{k_{1}}{\rho_{1}}\left(\partial_{x} v_{1}+v_{2}\right)_{x} \\
\quad+\frac{g_{0}}{\rho_{1}}\left(a \partial_{x} v_{1}\right)_{x}-\frac{1}{\rho_{1}} \int_{0}^{+\infty} g(s)\left(a \partial_{x} v_{6}(s)\right)_{x} d s=f_{7} \\
v_{2}-\frac{k_{2}}{\rho_{2}} \partial_{x x} v_{2}+\frac{k_{1}}{\rho_{2}}\left(\partial_{x} v_{1}+v_{2}\right)=f_{2}+f_{4}
\end{array}\right.
$$

where

$$
f_{7}=\frac{1}{\rho_{1}}\left(\rho_{1}+b+\xi_{0}+d e^{-\tau}\right) f_{1}+f_{3}-\frac{\tau d e^{-\tau}}{\rho_{1}} \int_{0}^{1} e^{\tau y} f_{5}(y) d y .
$$

So, it is sufficient to prove that (3.10), with $v_{6}$ given in (3.8), has a solution $\left(v_{1}, v_{2}\right)$ satisfying (3.9),

$$
\partial_{x} v_{2}(0)=\partial_{x} v_{2}(L)=0
$$

and

$$
k_{1} \partial_{x x} v_{1}-g_{0} \partial_{x}\left(a \partial_{x} v_{1}\right)+\int_{0}^{+\infty} g(s) \partial_{x}\left(a \partial_{x} v_{6}(s)\right) d s \in L^{2}(] 0, L[),
$$

and then, we replace $v_{1}$ and $v_{2}$ in (3.5), (3.7) and (3.8) to get $V \in D(A)$ satisfying (3.4). Let $\left(v_{1}, v_{2}\right)$ satisfying (3.9)-(3.11). By multiplying the equations in (3.10) by $\rho_{1} w_{1}$ and $\rho_{2} w_{2}$, respectively, integrating their sum by parts on $] 0, L[$ and exploiting (3.8) and (3.11), we find that $\left(v_{1}, v_{2}\right)$ is a solution of the system

$$
L_{1}\left(\left(v_{1}, v_{2}\right),\left(w_{1}, w_{2}\right)\right)=L_{2}\left(w_{1}, w_{2}\right), \quad \forall\left(w_{1}, w_{2}\right) \in H_{0}^{1}(] 0, L[) \times H_{*}^{1}(] 0, L[),
$$

where

$$
\begin{aligned}
& L_{1}\left(\left(v_{1}, v_{2}\right),\left(w_{1}, w_{2}\right)\right)=\int_{0}^{L}\left(k_{1}\left(\partial_{x} v_{1}+v_{2}\right)\left(\partial_{x} w_{1}+w_{2}\right)+k_{2} \partial_{x} v_{2} \partial_{x} w_{2}\right) d x \\
& \quad+\int_{0}^{L}\left(-a g_{1} \partial_{x} v_{1} \partial_{x} w_{1}+\left(\rho_{1}+b+\xi_{0}+d e^{-\tau}\right) v_{1} w_{1}+\rho_{2} v_{2} w_{2}\right) d x \\
& L_{2}\left(\left(w_{1}, w_{2}\right)\right)=\int_{0}^{L}\left(\rho_{1} f_{7} w_{1}+\partial_{x} f_{8} \partial_{x} w_{1}+\rho_{2}\left(f_{2}+f_{4}\right) w_{2}\right) d x \\
& g_{1}=\int_{0}^{+\infty} e^{-s} g(s) d s \text { and } f_{8}=a \int_{0}^{+\infty} e^{-s} g(s) \int_{0}^{s} e^{y}\left(f_{1}-f_{6}(y)\right) d y d s
\end{aligned}
$$

Since, it is easy to prove that $L_{1}$ is a bilinear, continuous and coercive form and $L_{2}$ is a linear and continuous form on, respectively,

$$
\left(H_{0}^{1}(] 0, L[) \times H_{*}^{1}(] 0, L[)\right)^{2} \text { and } H_{0}^{1}(] 0, L[) \times H_{*}^{1}(] 0, L[)
$$

(noting that $g_{1}<g_{0}$ and using (2.14)), so, applying the Lax-Milgram theorem, we deduce that (3.13) admits a unique solution

$$
\left(v_{1}, v_{2}\right) \in H_{0}^{1}(] 0, L[) \times H_{*}^{1}(] 0, L[) .
$$


Applying the classical elliptic regularity, it follows that $\left(v_{1}, v_{2}\right)$ satisfies (3.9)-(3.12). Therefore, the operator $I d+A$ is surjective.

Third, we see that the linear operator $B$ is Lipschitz continuous.

Because $-A$ is dissipative and $I d+A$ is surjective, then $A$ is a maximal monotone operator. Therefore, using Lummer-Phillips theorem (see [53]), we deduce that $A$ is an infinitesimal generator of a linear $C_{0}$-semigroup on $\mathcal{H}$. Finally, also $A+B$ is an infinitesimal generator of a linear $C_{0}$-semigroup on $\mathcal{H}$ (see [53]: Ch. 3-Theorem 1.1). Consequently, Theorem 2.3 holds from the Hille-Yosida theorem (see $[33,53]$ ).

\section{Some needed lemmas}

We will use $c$ (sometimes $c_{y}, c_{y, y_{1}}, \ldots$, which depends on some parameters $y, y_{1}, \ldots$ ), throughout the rest of this paper, to denote a generic positive constant which depends continuously on the initial data $U_{0}$ and can be different from step to step, but it does not depend neither on $b$ nor on $d$.

To get our stability results, we prove first some needed lemmas, for all $U_{0} \in D(A)$; so all the calculations are justified. By a simple density arguments $(D(A)$ is dense in $\mathcal{H}),(2.34)$ and (2.37) remain valid for any $U_{0} \in \mathcal{H}$. The first next seven lemmas, used in [25], are adapted in the present paper to (1.1) by considering the needed modifications related to the presence of delay.

We start by giving the following estimates for the derivative of $E$ :

Lemma 4.1 The energy functional satisfies, if (1.11) holds and $d \neq 0$,

$$
E^{\prime}(t) \leq \frac{1}{2} g^{\prime} \circ \varphi_{x}-\frac{1}{2} \inf _{[0, L]}(b-|d|) \int_{0}^{L} \varphi_{t}^{2} d x,
$$

and, if (1.12) holds or $d \equiv 0$,

$$
E^{\prime}(t) \leq \frac{1}{2} g^{\prime} \circ \varphi_{x}+\int_{0}^{L}\left(-b+\|d\|_{\infty}\right) \varphi_{t}^{2} d x .
$$

Proof By exploiting (2.29), (3.2) and the definition (2.27) of $B$, we obtain

$$
E^{\prime}(t) \leq \frac{1}{2} g^{\prime} \circ \varphi_{x}+\int_{0}^{L}\left(-b+\frac{\xi}{2 \tau}+\frac{|d|}{2}\right) \varphi_{t}^{2} d x+\int_{0}^{L}\left(\frac{|d|}{2}-\frac{\xi}{2 \tau}\right) \varphi_{t}^{2}(t-\tau) d x .
$$

So, from (2.25), we see that, if (1.11) holds and $d \neq 0$, then

$$
-b+\frac{\xi}{2 \tau}+\frac{|d|}{2}=\frac{|d|}{2}-\frac{\xi}{2 \tau}=-\frac{1}{2}(b-|d|) \leq-\frac{1}{2} \inf _{[0, L]}(b-|d|) \leq 0 .
$$

However, if (1.12) holds or $d \equiv 0$, we have

$-b+\frac{\xi}{2 \tau}+\frac{|d|}{2}=-b+\frac{\|d\|_{\infty}+|d|}{2} \leq-b+\|d\|_{\infty}$ and $\frac{|d|}{2}-\frac{\xi}{2 \tau}=\frac{|d|-\|d\|_{\infty}}{2} \leq 0$.

Hence, (4.3) yields (4.1) and (4.2).

Remark 4.2 1. When (1.11) holds, $E^{\prime} \leq 0$, since $g$ is non-increasing, and then (1.1) is dissipative. However, when (1.12) holds, we are unable to determine the sign of $E^{\prime}$ from (4.2), and therefore, (1.1) is not necessarily dissipative with respect to $E$ at this stage. 
2. Using the definition of $E$, (4.1) and (4.2), we see that, for some non-negative constant $\alpha_{0}, E^{\prime} \leq \alpha_{0} E$. Then, by integrating,

$$
E(t) \leq e^{\alpha_{0}\left(t-t_{0}\right)} E\left(t_{0}\right), \quad \forall t \geq t_{0} \geq 0 .
$$

So, if $E\left(t_{0}\right)=0$, for some $t_{0} \in \mathbb{R}_{+}$, then $E(t)=0$, for all $t \geq t_{0}$, and therefore, (2.34), (2.37) and (2.41) hold. Consequently, without loss of generality, we can assume that $E(t)>0$, for all $t \in \mathbb{R}_{+}$.

Lemma 4.3 The following inequalities hold:

$$
\begin{aligned}
& \exists d_{1}>0:\left(\int_{0}^{L} a \int_{0}^{+\infty} g(s)(\varphi(t)-\varphi(t-s)) d s d x\right)^{2} \leq d_{1} g \circ \varphi_{x} \\
& \exists d_{2}>0:\left(\int_{0}^{L} a \int_{0}^{+\infty} g^{\prime}(s)(\varphi(t)-\varphi(t-s)) d s d x\right)^{2} \leq-d_{2} g^{\prime} \circ \varphi_{x} \\
& \exists d_{3}>0:\left(\int_{0}^{L} a^{\prime} \int_{0}^{+\infty} g(s)(\varphi(t)-\varphi(t-s)) d s d x\right)^{2} \leq d_{3} g \circ \varphi_{x} \\
& \left(\int_{0}^{+\infty} g(s)\left(\varphi_{x}(t)-\varphi_{x}(t-s)\right) d s\right)^{2} \leq g_{0} \int_{0}^{+\infty} g(s)\left(\varphi_{x}(t)-\varphi_{x}(t-s)\right)^{2} d s \\
& \left(\int_{0}^{+\infty} g^{\prime}(s)\left(\varphi_{x}(t)-\varphi_{x}(t-s)\right) d s\right)^{2} \leq-g(0) \int_{0}^{+\infty} g^{\prime}(s)\left(\varphi_{x}(t)-\varphi_{x}(t-s)\right)^{2} d s
\end{aligned}
$$

Proof If $a \equiv 0,(4.4)-(4.6)$ are trivial. If $\inf _{[0, L]} a>0$, we use the fact that $a$ and $a^{\prime}$ are bounded and apply Poincaré's and Hölder's inequalities (2.26) (for $\varphi$ ) and

$$
\left(\int_{0}^{L}\left|f_{1} f_{2}\right| d x\right)^{2} \leq\left(\int_{0}^{L} f_{1}^{2} d x\right)\left(\int_{0}^{L} f_{2}^{2} d x\right), \quad \forall f_{1}, f_{2} \in L^{2}(] 0, L[),
$$

respectively, to get (4.4)-(4.6). Using again Hölder's inequality (4.9), (4.7) and (4.8) hold. Notice that the constants $d_{i}$ do not depend neither on $b$ nor on $d$.

\section{Lemma 4.4 The functional}

$$
I_{1}(t):=-\rho_{1} \int_{0}^{L} a \varphi_{t} \int_{0}^{+\infty} g(s)(\varphi(t)-\varphi(t-s)) d s d x
$$

satisfies, for any $\delta>0$,

$$
\begin{aligned}
I_{1}^{\prime}(t) \leq & -\rho_{1} g_{0} \int_{0}^{L} a \varphi_{t}^{2} d x+\delta \int_{0}^{L}\left(\varphi_{t}^{2}+\varphi_{x}^{2}+\psi_{x}^{2}\right) d x \\
& +\delta \int_{0}^{L}\left(b^{2} \varphi_{t}^{2}+d^{2} \varphi_{t}^{2}(t-\tau)\right) d x+c\left(1+\frac{1}{\delta}\right) g \circ \varphi_{x}-\frac{c}{\delta} g^{\prime} \circ \varphi_{x} .
\end{aligned}
$$

Proof First, note that

$$
\begin{aligned}
& \partial_{t}\left(\int_{0}^{+\infty} g(s)(\varphi(t)-\varphi(t-s)) d s\right)=\partial_{t}\left(\int_{-\infty}^{t} g(t-s)(\varphi(t)-\varphi(s)) d s\right) \\
& \quad=\int_{-\infty}^{t} g(t-s) \varphi_{t}(t) d s+\int_{-\infty}^{t} g^{\prime}(t-s)(\varphi(t)-\varphi(s)) d s \\
& =g_{0} \varphi_{t}+\int_{0}^{+\infty} g^{\prime}(s)(\varphi(t)-\varphi(t-s)) d s .
\end{aligned}
$$


Then, by differentiating $I_{1}$, and using the first equation and the boundary conditions in (1.1), we find

$$
\begin{aligned}
I_{1}^{\prime}(t)= & -\rho_{1} g 0 \int_{0}^{L} a \varphi_{t}^{2} d x-\rho_{1} \int_{0}^{L} a \varphi_{t} \int_{0}^{+\infty} g^{\prime}(s)(\varphi(t)-\varphi(t-s)) d s d x \\
& +k_{1} \int_{0}^{L} a\left(\varphi_{x}+\psi\right) \int_{0}^{+\infty} g(s)\left(\varphi_{x}(t)-\varphi_{x}(t-s)\right) d s d x \\
& +\int_{0}^{L} a\left(b \varphi_{t}+d \varphi_{t}(t-\tau)\right) \int_{0}^{+\infty} g(s)(\varphi(t)-\varphi(t-s)) d s d x \\
& +\int_{0}^{L} a^{2}\left(\int_{0}^{+\infty} g(s)\left(\varphi_{x}(t)-\varphi_{x}(t-s)\right) d s\right)^{2} d x-g_{0} \int_{0}^{L} a^{2} \varphi_{x} \int_{0}^{+\infty} g(s)\left(\varphi_{x}(t)\right. \\
& \left.-\varphi_{x}(t-s)\right) d s d x+\int_{0}^{L} a a^{\prime}\left(\int_{0}^{+\infty} g(s)(\varphi(t)-\varphi(t-s)) d s\right) \\
& \times\left(\int_{0}^{+\infty} g(s)\left(\varphi_{x}(t)-\varphi_{x}(t-s)\right) d s\right) d x \\
& +k_{1} \int_{0}^{L} a^{\prime}\left(\varphi_{x}+\psi\right) \int_{0}^{+\infty} g(s)(\varphi(t)-\varphi(t-s)) d s d x \\
& -g_{0} \int_{0}^{L} a a^{\prime} \varphi_{x} \int_{0}^{+\infty} g(s)(\varphi(t)-\varphi(t-s)) d s d x .
\end{aligned}
$$

Therefore, applying Young's and Hölder's inequalities (3.1) and (4.9), for the last eight terms of the above equality, and using (4.4)-(4.7), Poincaré's inequality (2.26), for $\varphi$, and the fact that $a$ and $a^{\prime}$ are bounded, we get (4.11).

\section{Lemma 4.5 The functional}

$$
I_{2}(t):=\int_{0}^{L}\left(\rho_{1} \varphi \varphi_{t}+\rho_{2} \psi \psi_{t}\right) d x
$$

satisfies, for any $\delta>0$,

$$
\begin{aligned}
I_{2}^{\prime}(t) \leq & \int_{0}^{L}\left(\rho_{1} \varphi_{t}^{2}+\rho_{2} \psi_{t}^{2}\right) d x-k_{1} \int_{0}^{L}\left(\varphi_{x}+\psi\right)^{2} d x-k_{2} \int_{0}^{L} \psi_{x}^{2} d x \\
& +g_{0} \int_{0}^{L} a \varphi_{x}^{2} d x+\delta \int_{0}^{L} \varphi_{x}^{2} d x+\frac{c}{\delta} \int_{0}^{L}\left(b^{2} \varphi_{t}^{2}+d^{2} \varphi_{t}^{2}(t-\tau)\right) d x+\frac{c}{\delta} g \circ \varphi_{x} .
\end{aligned}
$$

Proof By differentiating $I_{2}$, and using the first two equations and boundary conditions in (1.1), we have

$$
\begin{aligned}
I_{2}^{\prime}(t)= & \int_{0}^{L}\left(\rho_{1} \varphi_{t}^{2}+\rho_{2} \psi_{t}^{2}\right) d x-k_{1} \int_{0}^{L}\left(\varphi_{x}+\psi\right)^{2} d x-k_{2} \int_{0}^{L} \psi_{x}^{2} d x \\
& +g_{0} \int_{0}^{L} a \varphi_{x}^{2} d x-\int_{0}^{L} \varphi\left(b \varphi_{t}+d \varphi_{t}(t-\tau)\right) d x \\
& -\int_{0}^{L} a \varphi_{x} \int_{0}^{+\infty} g(s)\left(\varphi_{x}(t)-\varphi_{x}(t-s)\right) d s d x .
\end{aligned}
$$

Consequently, aplying Young's and Hölder's inequalities (3.1) and (4.9), for the last two terms of the above equality, and using (4.7), Poincaré's inequality (2.26), for $\varphi$, and the fact that $a$ is bounded, we find (4.14).

\section{를 Springer}


Lemma 4.6 The functional

$$
\begin{aligned}
I_{3}(t):= & -\rho_{2} \int_{0}^{L} \psi_{t}\left(\varphi_{x}+\psi\right) d x-\frac{k_{2} \rho_{1}}{k_{1}} \int_{0}^{L} \psi_{x} \varphi_{t} d x \\
& +\frac{\rho_{2}}{k_{1}} \int_{0}^{L} a \psi_{t} \int_{0}^{+\infty} g(s) \varphi_{x}(t-s) d s d x
\end{aligned}
$$

satisfies, for any $\delta, \delta_{1}>0$,

$$
\begin{aligned}
I_{3}^{\prime}(t) \leq & k_{1} \int_{0}^{L}\left(\varphi_{x}+\psi\right)^{2} d x-\rho_{2} \int_{0}^{L} \psi_{t}^{2} d x \\
& +g_{0}\left(\frac{\delta_{1}}{2}-1\right) \int_{0}^{L} a \varphi_{x}^{2} d x+\frac{g_{0} k_{0}\|a\|_{\infty}}{2 \delta_{1}} \int_{0}^{L} \psi_{x}^{2} d x \\
& +\frac{c}{\delta} \int_{0}^{L}\left(b^{2} \varphi_{t}^{2}+d^{2} \varphi_{t}^{2}(t-\tau)\right) d x \\
& +\delta \int_{0}^{L}\left(\psi_{t}^{2}+\varphi_{x}^{2}+\psi_{x}^{2}\right) d x+\frac{c}{\delta}\left(g \circ \varphi_{x}-g^{\prime} \circ \varphi_{x}\right) \\
& +\left(\frac{k_{2} \rho_{1}}{k_{1}}-\rho_{2}\right) \int_{0}^{L} \varphi_{x t} \psi_{t} d x
\end{aligned}
$$

where $k_{0}$ is defined in (2.5).

Proof Similarly to (4.12) and using that $\lim _{s \rightarrow+\infty} g(s)=0$, we see that

$$
\begin{aligned}
\partial_{t}\left(\int_{0}^{+\infty} g(s) \varphi_{x}(t-s) d s\right) & =\partial_{t}\left(\int_{-\infty}^{t} g(t-s) \varphi_{x}(s) d s\right) \\
& =g(0) \varphi_{x}+\int_{-\infty}^{t} g^{\prime}(t-s) \varphi_{x}(s) d s \\
& =g(0) \varphi_{x}+\int_{0}^{+\infty} g^{\prime}(s)\left(\varphi_{x}(t-s)-\varphi_{x}(t)+\varphi_{x}(t)\right) d s \\
& =-\int_{0}^{+\infty} g^{\prime}(s)\left(\varphi_{x}(t)-\varphi_{x}(t-s)\right) d s .
\end{aligned}
$$

Therefore, exploiting the first two equations and boundary conditions in (1.1), we have

$$
\begin{aligned}
I_{3}^{\prime}(t)= & k_{1} \int_{0}^{L}\left(\varphi_{x}+\psi\right)^{2} d x-\rho_{2} \int_{0}^{L} \psi_{t}^{2} d x+\left(\frac{k_{2} \rho_{1}}{k_{1}}-\rho_{2}\right) \int_{0}^{L} \varphi_{x t} \psi_{t} d x \\
& -g_{0} \int_{0}^{L} a \varphi_{x}^{2} d x-g_{0} \int_{0}^{L} a \varphi_{x} \psi d x+\int_{0}^{L} a\left(\varphi_{x}+\psi\right) \int_{0}^{+\infty} g(s)\left(\varphi_{x}(t)-\varphi_{x}(t-s)\right) d s d x \\
& -\frac{\rho_{2}}{k_{1}} \int_{0}^{L} a \psi_{t} \int_{0}^{+\infty} g^{\prime}(s)\left(\varphi_{x}(t)-\varphi_{x}(t-s)\right) d s d x+\frac{k_{2}}{k_{1}} \int_{0}^{L} \psi_{x}\left(b \varphi_{t}+d \varphi_{t}(t-\tau)\right) d x
\end{aligned}
$$

By applying Young's inequality (3.1), for the last four terms of the above equality, Poincaré's inequality (2.5), for $\psi$, and using (4.7), (4.8) and the fact that $a$ is bounded, (4.16) is established. 
Now, as in [7], we use a function $w$ to get a crucial estimate.

\section{Lemma 4.7 The function}

$$
w(x, t)=\int_{0}^{x} \psi(y, t) d y
$$

satisfies the estimates ( $\tilde{k}_{0}$ is the constant defined in (2.26))

$$
\begin{aligned}
& \int_{0}^{L} w_{x}^{2} d x=\int_{0}^{L} \psi^{2} d x, \quad \forall t \geq 0, \\
& \int_{0}^{L} w_{t}^{2} d x \leq \tilde{k}_{0} \int_{0}^{L} \psi_{t}^{2} d x, \quad \forall t \geq 0 .
\end{aligned}
$$

Proof We just have to note that $w_{x}=\psi$ to get (4.18). On the other hand, using (2.12) (remind that we are working with $\tilde{\psi}$, but we use $\psi$ instead of $\tilde{\psi}$; see Remark 2.1-2),

$$
w_{t}(0, t)=0 \text { and } w_{t}(L, t)=\int_{0}^{L} \psi_{t}(y, t) d y=\partial_{t} \int_{0}^{L} \psi(y, t) d y=0 .
$$

Then, applying (4.18) to $w_{t}$ and using Poincaré's inequality (2.26), for $w_{t}$, we arrive at (4.19).

\section{Lemma 4.8 The functional}

$$
I_{4}(t):=\rho_{1} \int_{0}^{L}\left(w \varphi_{t}+\varphi \varphi_{t}\right) d x
$$

satisfies, for any $\delta, \epsilon, \epsilon_{1}>0$,

$$
\begin{aligned}
I_{4}^{\prime}(t) \leq & \left(\rho_{1}+\frac{c_{0}}{\epsilon}\right) \int_{0}^{L} \varphi_{t}^{2} d x+c_{0} \epsilon \int_{0}^{L} \psi_{t}^{2} d x \\
& +\left(g_{0}\|a\|_{\infty}\left(1+\frac{\epsilon_{1}}{2}\right)-k_{1}\right) \int_{0}^{L}\left(\varphi_{x}+\psi\right)^{2} d x+\frac{g_{0} k_{0}\|a\|_{\infty}}{2 \epsilon_{1}} \int_{0}^{L} \psi_{x}^{2} d x \\
& +\delta \int_{0}^{L}\left(\varphi_{x}^{2}+\psi_{x}^{2}\right) d x+\frac{c}{\delta} \int_{0}^{L}\left(b^{2} \varphi_{t}^{2}+d^{2} \varphi_{t}^{2}(t-\tau)\right) d x+\frac{c}{\delta} g \circ \varphi_{x}
\end{aligned}
$$

where $k_{0}$ is defined in (2.5), $c_{0}=\frac{\rho_{1}}{2} \sqrt{\tilde{k}_{0}}$ and $\tilde{k}_{0}$ is defined in (2.26).

Proof Using the first two equations and boundary conditions in (1.1), and exploiting the fact that $w(0, t)=w(L, t)=0$ and $w_{x}=\psi$, we find

$$
\begin{aligned}
I_{4}^{\prime}(t)= & \rho_{1} \int_{0}^{L} \varphi_{t}^{2} d x-k_{1} \int_{0}^{L}\left(\varphi_{x}+\psi\right)^{2} d x \\
& +g_{0} \int_{0}^{L} a\left(\varphi_{x}+\psi-\psi\right)\left(\varphi_{x}+\psi\right) d x+\rho_{1} \int_{0}^{L} w_{t} \varphi_{t} d x \\
& -\int_{0}^{L}(w+\varphi)\left(b \varphi_{t}+d \varphi_{t}(t-\tau)\right) d x-\int_{0}^{L} a\left(\varphi_{x}+\psi\right) \int_{0}^{+\infty} g(s)\left(\varphi_{x}(t)\right. \\
& \left.-\varphi_{x}(t-s)\right) d s d x .
\end{aligned}
$$

Applying Young's inequality (3.1), for the last four terms of the above equality, Poincaré's inequalities (2.5), for $\psi$, and (2.26), for $\varphi$ and $w$, and exploiting (4.7), (4.18), (4.19) and the fact that $a$ is bounded, we get (4.21).

\section{Springer}


We use a functional introduced in [48] (in case $\xi \equiv 1$ ) to get an estimation on the delay term.

\section{Lemma 4.9 The functional}

$$
I_{5}(t)=\int_{0}^{L} \xi \int_{0}^{1} e^{-2 \tau p} \varphi_{t}^{2}(t-\tau p) d p d x
$$

satisfies

$$
\begin{aligned}
I_{5}^{\prime}(t) \leq & -2 e^{-2 \tau} \int_{0}^{L} \xi \int_{0}^{1} \varphi_{t}^{2}(t-\tau p) d p d x \\
& +\frac{1}{\tau} \int_{0}^{L} \xi \varphi_{t}^{2} d x-\frac{e^{-2 \tau}}{\tau} \int_{0}^{L} \xi \varphi_{t}^{2}(t-\tau) d x .
\end{aligned}
$$

Proof Using (2.16) and the first equation in (2.18), the derivative of $I_{5}$ entails

$$
\begin{aligned}
I_{5}^{\prime}(t) & =2 \int_{0}^{L} \xi \int_{0}^{1} e^{-2 \tau p} \varphi_{t t}(t-\tau p) \varphi_{t}(x, t-\tau p) d p d x \\
& =-\frac{2}{\tau} \int_{0}^{L} \xi \int_{0}^{1} e^{-2 \tau p} \varphi_{t p}(t-\tau p) \varphi_{t}(t-\tau p) d p d x \\
& =-\frac{1}{\tau} \int_{0}^{L} \xi \int_{0}^{1} e^{-2 \tau p} \partial_{p} \varphi_{t}^{2}(t-\tau p) d p d x .
\end{aligned}
$$

Then, by using an integrating by parts, the above formula can be rewritten as

$I_{5}^{\prime}(t)=-2 \int_{0}^{L} \xi \int_{0}^{1} e^{-2 \tau p} \varphi_{t}^{2}(t-\tau p) d p d x+\frac{1}{\tau} \int_{0}^{L} \xi \varphi_{t}^{2} d x-\frac{e^{-2 \tau}}{\tau} \int_{0}^{L} \xi \varphi_{t}^{2}(t-\tau) d x$,

which gives (4.23), since $-2 e^{-2 \tau p} \leq-2 e^{-2 \tau}$, for any $\left.p \in\right] 0,1[$.

Let $a_{0}:=\inf _{[0, L]} a, b_{0}:=\inf _{[0, L]} b$ and, for $N, N_{1}, N_{2}, N_{3}, N_{4} \geq 0$,

$$
I_{6}:=N E+N_{1} I_{1}+N_{2} I_{2}+I_{3}+N_{3} I_{4}+N_{4} I_{5} \text {. }
$$

Then, by combining (4.11), (4.14), (4.16), (4.21) and (4.23), we obtain

$$
\begin{aligned}
I_{6}^{\prime}(t) \leq & -\int_{0}^{L}\left(l_{0} \varphi_{t}^{2}+l_{1} \psi_{t}^{2}+l_{2}\left(\varphi_{x}+\psi\right)^{2}+l_{3} \psi_{x}^{2}\right) d x+l_{4} g_{0} \int_{0}^{L} a \varphi_{x}^{2} d x+N E^{\prime}(t) \\
& -2 e^{-2 \tau} N_{4} \int_{0}^{L} \xi \int_{0}^{1} \varphi_{t}^{2}(t-\tau p) d p d x+\delta\left(N_{1}+c_{N_{2}, N_{3}}\right) \int_{0}^{L}\left(\varphi_{t}^{2}+\psi_{t}^{2}+\varphi_{x}^{2}+\psi_{x}^{2}\right) d x \\
& -\int_{0}^{L}\left(\frac{e^{-2 \tau} N_{4}}{\tau} \xi-\left(\delta N_{1}+\frac{c_{N_{2}, N_{3}}}{\delta}\right) d^{2}\right) \varphi_{t}^{2}(t-\tau) d x \\
& +\int_{0}^{L}\left(\frac{N_{4}}{\tau} \xi+\left(\delta N_{1}+\frac{c_{N_{2}, N_{3}}}{\delta}\right) b^{2}\right) \varphi_{t}^{2} d x \\
& +\left(c_{N_{1}}+\frac{c_{N_{1}, N_{2}, N_{3}}}{\delta}\right) g \circ \varphi_{x}-\frac{c_{N_{1}}}{\delta} g^{\prime} \circ \varphi_{x}+\left(\frac{\rho_{1} k_{2}}{k_{1}}-\rho_{2}\right) \int_{0}^{L} \varphi_{x t} \psi_{t} d x,
\end{aligned}
$$

where

$$
\begin{aligned}
& l_{0}=N_{1} \rho_{1} g_{0} a_{0}-\left(N_{2}+N_{3}\right) \rho_{1}-\frac{c_{0} N_{3}}{\epsilon}, \\
& l_{1}=\rho_{2}\left(1-N_{2}\right)-c_{0} \epsilon N_{3}, \quad l_{2}=k_{1}\left(N_{2}+N_{3}-1\right)-g_{0}\|a\|_{\infty}\left(1+\frac{\epsilon_{1}}{2}\right) N_{3}, \\
& l_{3}=k_{2} N_{2}-\frac{g_{0} k_{0}\|a\|_{\infty}}{2}\left(\frac{N_{3}}{\epsilon_{1}}+\frac{1}{\delta_{1}}\right) \text { and } l_{4}=N_{2}+\frac{\delta_{1}}{2}-1 .
\end{aligned}
$$


Now, as in [25], we choose carefully the constants $N, N_{i}, \delta, \delta_{1}, \epsilon$ and $\epsilon_{1}$ to get desired signs of $l_{i}$.

Case $1 a \equiv 0$ : the second integral in (4.25) drops, $g \circ \varphi_{x}=g^{\prime} \circ \varphi_{x}=0$ (from the definition (2.32)) and the constants $l_{0}, l_{1}, l_{2}$ and $l_{3}$ do not depent neither on $\delta_{1}$ nor on $\epsilon_{1}$. On the other hand,

$$
l_{0}=-\left(N_{2}+N_{3}\right) \rho_{1}-\frac{c_{0} N_{3}}{\epsilon} \geq N_{1} b_{0}-\left(N_{2}+N_{3}\right) \rho_{1}-\frac{c_{0} N_{3}}{\epsilon}-N_{1} b:=\tilde{l}_{0}-N_{1} b,
$$

so $\tilde{l}_{0}:=N_{1} b_{0}-\left(N_{2}+N_{3}\right) \rho_{1}-\frac{c_{0} N_{3}}{\epsilon}$. Therefore, we choose

$$
N_{3}=1, \quad 0<N_{2}<1, \quad 0<\epsilon<\frac{\rho_{2}}{c_{0}}\left(1-N_{2}\right) \quad \text { and } \quad N_{1}>\frac{1}{b_{0}}\left(N_{2}+N_{3}\right)+\frac{c_{0} N_{3}}{\epsilon b_{0}} .
$$

Notice that $N_{3}, N_{2}$ and $\epsilon$ do not depend neither on $b$ nor on $d$. Moreover, because $b_{0}>0$ thanks to (2.2) and $a \equiv 0, N_{1}$ exists and can be taken in the form $N_{1}=\frac{c}{b_{0}}$, and then $\tilde{l}_{0}$ as well as $l_{1}, l_{2}$ and $l_{3}$ do not depend neither on $b$ nor on $d$. According to these choices, we get

$$
L:=\min \left\{\frac{\tilde{l}_{0}}{\rho_{1}}, \frac{l_{1}}{\rho_{2}}, \frac{l_{2}}{k_{1}}, \frac{l_{3}}{k_{2}}\right\}>0,
$$

and then, using (2.14) and (4.25),

$$
\begin{aligned}
I_{6}^{\prime}(t) \leq & -\left(L-c \delta\left(1+\frac{1}{b_{0}}\right)\right) \int_{0}^{L}\left(\rho_{1} \varphi_{t}^{2}+\rho_{2} \psi_{t}^{2}+k_{1}\left(\varphi_{x}+\psi\right)^{2}\right. \\
& \left.+k_{2} \psi_{x}^{2}\right) d x-2 e^{-2 \tau} N_{4} \int_{0}^{L} \xi \int_{0}^{1} \varphi_{t}^{2}(t-\tau p) d p d x \\
& -\int_{0}^{L}\left(\frac{e^{-2 \tau} N_{4}}{\tau} \xi-c\left(\frac{\delta}{b_{0}}+\frac{1}{\delta}\right) d^{2}\right) \varphi_{t}^{2}(t-\tau) d x \\
& +N E^{\prime}(t)+\int_{0}^{L}\left(\frac{N_{4}}{\tau} \xi+c\left(\frac{b^{2} \delta}{b_{0}}+\frac{b^{2}}{\delta}+\frac{b}{b_{0}}\right)\right) \varphi_{t}^{2} d x \\
& +\left(\frac{\rho_{1} k_{2}}{k_{1}}-\rho_{2}\right) \int_{0}^{L} \varphi_{x t} \psi_{t} d x .
\end{aligned}
$$

Next, choosing $\delta>0$ such that

$$
L-c \delta\left(1+\frac{1}{b_{0}}\right)>0 .
$$

Notice that $L$ and $c$ do not depend on $\delta, b$ and $d$; so $\delta$ exists and can be taken in the form

$$
\delta=\frac{c b_{0}}{b_{0}+1}
$$

and consequently, $L-c \delta\left(1+\frac{1}{b_{0}}\right)$ is a positive constant which does not depend neither on $b$ nor on $d$. At the end, we choose $N_{4}$ such that

$$
\frac{e^{-2 \tau} N_{4}}{\tau} \xi-c\left(\frac{\delta}{b_{0}}+\frac{1}{\delta}\right) d^{2} \geq 0 .
$$

If $d \equiv 0$, then $\xi \equiv 0$ (thanks to (2.25)), and therefore (4.28) is satisfied, for any $N_{4} \geq 0$. Otherwise, if $d \neq 0$, then $\xi=\tau b$ (in vertue of (2.25) and because (1.11) is assumed in this

\section{Springer}


case $a \equiv 0$; see Theorem 2.6), consequently, the choice (4.27) and the inequality $|d|<b$ (according to (1.11)) imply that $N_{4}$ can be taken in the form

$$
N_{4}=\frac{c\|b\|_{\infty}\left(b_{0}+1\right)}{b_{0}} .
$$

Thus, using (2.31), we get from (4.26)

$$
\begin{aligned}
I_{6}^{\prime}(t) \leq & -c E_{0}(t)-\frac{c\|b\|_{\infty}\left(b_{0}+1\right)}{b_{0}} E_{1}(t)+N E^{\prime}(t) \\
& +\frac{c\left(\|b\|_{\infty}\left(b_{0}+1\right)+1\right)}{b_{0}} \int_{0}^{L} b \varphi_{t}^{2} d x+\left(\frac{\rho_{1} k_{2}}{k_{1}}-\rho_{2}\right) \int_{0}^{L} \varphi_{x t} \psi_{t} d x,
\end{aligned}
$$

where

$$
E_{0}(t)=E(t)-E_{1}(t) \text { and } E_{1}(t)=\frac{1}{2} \int_{0}^{L} \xi \int_{0}^{1} \varphi_{t}^{2}(t-\tau p) d p d x .
$$

Case 2. $a_{0}>0$ : we choose

$$
\epsilon_{1}=\frac{k_{1}-g_{0}\|a\|_{\infty}}{g_{0}\|a\|_{\infty}}, \quad \delta_{1}=\frac{k_{0} g_{0}\|a\|_{\infty}}{k_{2}},
$$

$$
\frac{k_{1} \delta_{1}}{2 k_{1}-g_{0}\|a\|_{\infty}\left(2+\epsilon_{1}\right)}<N_{3}<\epsilon_{1}\left(\frac{k_{2}\left(2-\delta_{1}\right)}{g_{0} k_{0}\|a\|_{\infty}}-\frac{1}{\delta_{1}}\right)
$$

$$
\max \left\{1-N_{3}\left(1-\frac{g_{0}\|a\|_{\infty}\left(2+\epsilon_{1}\right)}{2 k_{1}}\right), \frac{g_{0} k_{0}\|a\|_{\infty}}{2 k_{2}}\left(\frac{N_{3}}{\epsilon_{1}}+\frac{1}{\delta_{1}}\right)\right\}<N_{2}<1-\frac{\delta_{1}}{4}
$$

$$
0<\epsilon<\min \left\{\left(2\left(1-N_{2}\right)-\frac{\delta_{1}}{2}\right) \frac{\rho_{2}}{c_{0} N_{3}}, \frac{\rho_{2}\left(1-N_{2}\right)}{c_{0} N_{3}}\right\}
$$

and

$$
N_{1}>\max \left\{\frac{\left(N_{2}+N_{3}\right) \rho_{1}+\frac{c_{0} N_{3}}{\epsilon}}{\rho_{1} g_{0} a_{0}}, \frac{\left(2 N_{2}+N_{3}+\frac{\delta_{1}}{2}-1\right) \rho_{1}+\frac{c_{0} N_{3}}{\epsilon}}{\rho_{1} g_{0} a_{0}}\right\} .
$$

Notice that $\epsilon_{1}$ and $\delta_{1}$ exist and are positive thanks to (2.4) and the property $g_{0}>0$ (because $g(0)>0$; see $(\mathrm{H} 2)), N_{2}$ exists according to the choice of $N_{3}, \epsilon$ exists from the choice of $N_{2}$, and $N_{1}$ exists because $\rho_{1} g_{0} a_{0}>0$. On the other hand, to prove the existence of $N_{3}$, we repeat the calculations given in [25]. Using the definitions of $\epsilon_{1}$ and $\delta_{1}$, we see that $N_{3}$ exists if and only if

$$
k_{0}^{2} k_{1}\left(g_{0}\|a\|_{\infty}\right)^{3}<k_{2}\left(k_{2}-k_{0} g_{0}\|a\|_{\infty}\right)\left(k_{1}-g_{0}\|a\|_{\infty}\right)^{2} .
$$

Let $\left.y_{0}=\frac{k_{1} k_{2}}{k_{0} k_{1}+k_{2}}, y=g_{0}\|a\|_{\infty} \in\right] 0, y_{0}[$ (see (2.4)) and

$$
f(y)=k_{0}^{2} k_{1} y^{3}-k_{2}\left(k_{2}-k_{0} y\right)\left(k_{1}-y\right)^{2} .
$$

We have

$$
f^{\prime}(y)=3\left(k_{0}^{2} k_{1}+k_{0} k_{2}\right) y^{2}-2\left(2 k_{0} k_{1} k_{2}+k_{2}^{2}\right) y+k_{0} k_{1}^{2} k_{2}+2 k_{1} k_{2}^{2}
$$


and

$$
f^{\prime \prime}(y)=6\left(k_{0}^{2} k_{1}+k_{0} k_{2}\right) y-2\left(2 k_{0} k_{1} k_{2}+k_{2}^{2}\right) .
$$

Let $y_{1}=\frac{2 k_{0} k_{1} k_{2}+k_{2}^{2}}{3\left(k_{0}^{2} k_{1}+k_{0} k_{2}\right)}$. We notice that $f^{\prime}$ is decreasing on $] 0, y_{1}[$, it is increasing on $] y_{1},+\infty[$ and

$$
f^{\prime}\left(y_{0}\right)=\frac{k_{0}^{2} k_{1}^{3} k_{2}+2 k_{0} k_{1}^{2} k_{2}^{2}}{k_{0} k_{1}+k_{2}}>0,
$$

Moreover, $y_{1} \leq y_{0}$ if and only if $k_{2} \leq k_{0} k_{1}$, and, if $k_{2} \leq k_{0} k_{1}$,

$$
f^{\prime}\left(y_{1}\right)=\frac{5 k_{0}^{2} k_{1}^{2} k_{2}^{2}-k_{2}^{4}+2 k_{0} k_{1} k_{2}^{3}+3 k_{0}^{3} k_{1}^{3} k_{2}}{3\left(k_{0}^{2} k_{1}+k_{0} k_{2}\right)} \geq \frac{9 k_{2}^{4}}{3\left(k_{0}^{2} k_{1}+k_{0} k_{2}\right)}>0 .
$$

Therefore, $f^{\prime}$ is positive on $] 0, y_{0}\left[\right.$, and then $f(y)<f\left(y_{0}\right)$, for any $\left.y \in\right] 0, y_{0}[$. But $f\left(y_{0}\right)=0$, hence $f$ is negative on $] 0, y_{0}\left[\right.$. This guarantees the existence of $N_{3}$.

By vertue of these choices, we notice that

$$
L:=\min \left\{\frac{l_{0}}{\rho_{1}}, \frac{l_{1}}{\rho_{2}}, \frac{l_{2}}{k_{1}}, \frac{l_{3}}{k_{2}}\right\}>0, \quad l_{4} \leq L,
$$

and $L$ does not depend on $\delta, b$ and $d$. Then, using (2.14) and (4.25), we find

$$
\begin{aligned}
I_{6}^{\prime}(t) \leq & -(L-c \delta) \int_{0}^{L}\left(\rho_{1} \varphi_{t}^{2}+\rho_{2} \psi_{t}^{2}+k_{1}\left(\varphi_{x}+\psi\right)^{2}+k_{2} \psi_{x}^{2}-a g_{0} \varphi_{x}^{2}\right) d x+N E^{\prime}(t) \\
& -2 e^{-2 \tau} N_{4} \int_{0}^{L} \xi \int_{0}^{1} \varphi_{t}^{2}(t-\tau p) d p d x-\int_{0}^{L}\left(\frac{e^{-2 \tau} N_{4}}{\tau} \xi-c\left(\delta+\frac{1}{\delta}\right) d^{2}\right) \varphi_{t}^{2}(t-\tau) d x \\
& +\int_{0}^{L}\left(\frac{N_{4}}{\tau} \xi+c\left(\delta+\frac{1}{\delta}\right) b^{2}\right) \varphi_{t}^{2} d x+c\left(1+\frac{1}{\delta}\right) g \circ \varphi_{x}-\frac{c}{\delta} g^{\prime} \circ \varphi_{x} \\
& +\left(\frac{\rho_{1} k_{2}}{k_{1}}-\rho_{2}\right) \int_{0}^{L} \varphi_{x t} \psi_{t} d x .
\end{aligned}
$$

Therefore, choosing $\delta>0$ and $N_{4} \geq 0$ such that $L-c \delta>0$ and

$$
\frac{e^{-2 \tau} N_{4}}{\tau} \xi-c\left(\delta+\frac{1}{\delta}\right) d^{2} \geq 0 .
$$

In vertue of (2.25), $N_{4}$ can be chosen in the form $N_{4}=c\|d\|_{\infty}$. Then, using (2.31), (4.30) and (4.32), we find, in both cases $a \equiv 0$ and $a_{0}>0$,

$$
\begin{aligned}
I_{6}^{\prime}(t) \leq & -c E_{0}(t)-\tilde{c} E_{1}(t)+N E^{\prime}(t) \\
& +c \int_{0}^{L} \tilde{\xi} \varphi_{t}^{2} d x+\left(\frac{\rho_{1} k_{2}}{k_{1}}-\rho_{2}\right) \int_{0}^{L} \varphi_{x t} \psi_{t} d x+c\left(g \circ \varphi_{x}-g^{\prime} \circ \varphi_{x}\right),
\end{aligned}
$$

where, thanks to the definition of $\xi$ in case (1.11),

$$
\tilde{c}= \begin{cases}\frac{c\|b\|_{\infty}\left(b_{0}+1\right)}{b_{0}} & \text { if } a \equiv 0 \\ c\|d\|_{\infty} & \text { if } a_{0}>0\end{cases}
$$

and

$$
\tilde{\xi}= \begin{cases}\frac{\|b\|_{\infty}\left(b_{0}+1\right)+1}{b_{0}} b & \text { if } a \equiv 0, \\ \|b\|_{\infty} b & \text { if } a_{0}>0 \text { and (1.11) holds, } \\ \|d\|_{\infty}^{2}+\|b\|_{\infty} b & \text { if } a_{0}>0 \text { and (1.12) holds. }\end{cases}
$$

\section{Springer}


Now, we estimate the term $g \circ \varphi_{x}$ in (4.33).

Case 1 (2.8) holds: then

$$
g \circ \varphi_{x} \leq-\frac{1}{\alpha} g^{\prime} \circ \varphi_{x} .
$$

Case 2 (2.9) holds: this case does not concern Theorem 2.6 because of (2.38). For Theorem 2.4 and Theorem 2.7, we apply here an inequality given in [19] (and in [16] in a less general form).

Lemma 4.10 For any $\epsilon_{0}>0$, we have

$$
G^{\prime}\left(\epsilon_{0} E(t)\right) g \circ \varphi_{x} \leq-c g^{\prime} \circ \varphi_{x}+c \epsilon_{0} E(t) G^{\prime}\left(\epsilon_{0} E(t)\right) .
$$

Proof In Theorem 2.4 and Theorem 2.7, it is assumed that (1.11) holds. Then, thanks to (4.1), $E$ is non-increasing. Therefore, the proof is the same as in [19]-Lemma 3.6 (for $B^{\frac{1}{2}}=\partial_{x}$ and $\left.\|\cdot\|=\|\cdot\|_{L^{2}(] 0, L[)}\right)$.

Using (4.33), (4.36) and (4.37), we see that, in both two previous cases,

$$
\begin{aligned}
& \frac{G_{0}(E(t))}{E(t)} I_{6}^{\prime}(t) \leq-\frac{G_{0}(E(t))}{E(t)}\left(\left(c-\tilde{\epsilon}_{0}\right) E_{0}(t)+\left(\tilde{c}-\tilde{\epsilon}_{0}\right) E_{1}(t)\right)+N \frac{G_{0}(E(t))}{E(t)} E^{\prime}(t) \\
& -c\left(1+G^{\prime}\left(\epsilon_{0} E(t)\right)\right) g^{\prime} \circ \varphi_{x}+c \frac{G_{0}(E(t))}{E(t)} \int_{0}^{L} \tilde{\xi} \varphi_{t}^{2} d x \\
& +\left(\frac{\rho_{1} k_{2}}{k_{1}}-\rho_{2}\right) \frac{G_{0}(E(t))}{E(t)} \int_{0}^{L} \varphi_{x t} \psi_{t} d x,
\end{aligned}
$$

where $G_{0}$ is defined in (2.35) and

$$
\tilde{\epsilon}= \begin{cases}0 & \text { if (2.8) holds, } \\ c \epsilon_{0} & \text { if (2.9) holds. }\end{cases}
$$

On the other hand, by (2.14) and the definitions of the functionals $I_{i}$ and $E$, there exists a positive constant $\beta$ (not depending on $N, b$ and $d$ ) satisfying

$$
\left|N_{1} I_{1}+N_{2} I_{2}+I_{3}+N_{3} I_{4}+N_{4} I_{5}\right| \leq \beta E,
$$

which implies that

$$
(N-\beta) E \leq I_{6} \leq(N+\beta) E .
$$

Now, at this stage, we distinguish the cases of Theorems 2.4, 2.6 and 2.7.

\section{General stability: (1.2) and (1.11) hold}

Using (4.1) (in case $d \neq 0$ ), (4.2) (in case $d \equiv 0$ ) and the property $g^{\prime} \leq 0$, we have

$$
N E^{\prime}(t)+c \int_{0}^{L} \tilde{\xi} \varphi_{t}^{2} d x \leq \int_{0}^{L}\left(c \tilde{\xi}-\frac{N}{2} \inf _{[0, L]}(b-|d|)\right) \varphi_{t}^{2} d x
$$

and

$$
-g^{\prime} \circ \varphi_{x} \leq-2 E^{\prime}(t)
$$


Therefore, inserting (5.1) and (5.2) into (4.38), choosing $\epsilon_{0}>0$ such that $\tilde{\epsilon}$ defined in (4.39) satisfies

$$
\tilde{\epsilon}< \begin{cases}\min \{c, \tilde{c}\} & \text { if } d \neq 0 \\ c & \text { if } d \equiv 0\end{cases}
$$

(if $d \equiv 0$, then $\xi=E_{1}=0$ and $E=E_{0}$ ) and choosing $N \geq 0$ such that

$$
c \tilde{\xi}-\frac{N}{2} \inf _{[0, L]}(b-|d|) \leq 0 \text { and } N>\beta
$$

( $N$ exists according to (1.11), (4.35) and the boundedness of $b$ ), we deduce, from (1.2), (4.38), (4.40) and the fact that $G^{\prime}\left(\epsilon_{0} E\right)$ is non-increasing, that $I_{6} \sim E$, the last term in (4.38) vanishes and, for some positive constant $\beta_{1}$,

$$
\frac{G_{0}(E(t))}{E(t)} I_{6}^{\prime}(t)+c E^{\prime}(t) \leq-\beta_{1} G_{0}(E(t)) .
$$

Let $\tau_{0}>0$ and

$$
F=\tau_{0}\left(\frac{G_{0}(E)}{E} I_{6}+c E\right)
$$

We have $F \sim E$ (because $I_{6} \sim E$ and $\frac{G_{0}(E)}{E}$ is non-increasing) and, using (5.3),

$$
F^{\prime} \leq-\tau_{0} \beta_{1} G_{0}(E)
$$

Then, for $\tau_{0}>0$ such that

$$
F \leq E \text { and } F(0) \leq 1
$$

we get, for $\alpha_{2}=\tau_{0} \beta_{1}>0$ (since $G_{0}$ is increasing),

$$
F^{\prime} \leq-\alpha_{2} G_{0}(F) .
$$

Then (5.7) implies that

$$
(\tilde{G}(F))^{\prime} \geq \alpha_{2},
$$

where $\tilde{G}(t)=\int_{t}^{1} \frac{1}{G_{0}(s)} d s$. Integrating (5.8) over $[0, t]$ yields

$$
\tilde{G}(F(t)) \geq \alpha_{2} t+\tilde{G}(F(0)) .
$$

Because $F(0) \leq 1, \tilde{G}(1)=0$ and $\tilde{G}$ is decreasing, we obtain from (5.9) that

$$
\tilde{G}(F(t)) \geq \alpha_{2} t
$$

which implies that

$$
F(t) \leq \tilde{G}^{-1}\left(\alpha_{2} t\right)
$$

The fact that $F \sim E$ gives (2.34). This completes the proof of Theorem 2.4.

\section{Springer}




\section{Exponential stability: (1.2) and (1.12) hold}

Exploiting (2.38), (4.2), (4.35) and the property $g^{\prime} \leq 0$, we find

$$
\begin{aligned}
& N E^{\prime}(t)+c \int_{0}^{L} \tilde{\xi} \varphi_{t}^{2} d x \leq \int_{0}^{L}\left(N\left(-b+\|d\|_{\infty}\right)+c\left(\|d\|_{\infty}^{2}+\|b\|_{\infty} b\right)\right) \varphi_{t}^{2} d x \\
& \leq \int_{0}^{L}\left(c\|b\|_{\infty}-N\right) b \varphi_{t}^{2} d x+\frac{2}{\rho_{1}}\left(N\|d\|_{\infty}+c\|d\|_{\infty}^{2}\right) E_{0}(t)
\end{aligned}
$$

and

$$
-g^{\prime} \circ \varphi_{x} \leq-2 E^{\prime}(t)+2\|d\|_{\infty} \int_{0}^{L} \varphi_{t}^{2} d x \leq-2 E^{\prime}(t)+\frac{4}{\rho_{1}}\|d\|_{\infty} E_{0}(t) .
$$

Therefore, choosing $N \geq 0$ such that

$$
N \geq c\|b\|_{\infty} \text { and } N>\beta
$$

so $c\|b\|_{\infty}-N \leq 0$ and $I_{6} \sim E$ by vertue of (4.40). The constant $N$ can be choosen in the form

$$
N=c\left(1+\|b\|_{\infty}\right),
$$

and therefore, inserting (6.1) and (6.2) into (4.38) and noting that the last term in (4.38) vanishes (thanks to (1.2)), $G_{0}=I d$ and $\tilde{\epsilon}_{0}=0$ (according to (2.35) and (4.39)), we conclude that, for some positive constant $\beta_{2}$ which does not depend neither on $b$ nor on $d$,

$$
I_{6}^{\prime}(t)+c E^{\prime}(t) \leq-\left(c-\beta_{2}\left(1+\|b\|_{\infty}\right)\left(\|d\|_{\infty}^{2}+\|d\|_{\infty}\right)\right) E_{0}(t)-\tilde{c} E_{1}(t) .
$$

Let $F=I_{6}+c E$. The property $I_{6} \sim E$ and condition (2.39), for

$$
d_{0}=\frac{c}{\beta_{2}\left(1+\|b\|_{\infty}\right)},
$$

lead to $F \sim E$ and

$$
F^{\prime} \leq-\alpha_{2} F
$$

for some positive constant $\alpha_{2}$. By integrating (6.5) over $[0, t]$ and using again the equivalence $F \sim E$, we find (2.37). This ends the proof of Theorem 2.6.

\section{Weak stability: (1.2) does not hold and (1.11) holds}

In this section, we treat the case when (1.2) does not hold which is more realistic from the physics point of view. We need to estimate the last term in (4.38) using the system (7.1) resulting from differentiating (1.1) with respect to time

$$
\left\{\begin{array}{l}
\rho_{1} \varphi_{t t t}-k_{1}\left(\varphi_{x t}+\psi_{t}\right)_{x}+d \varphi_{t t}(t-\tau)+b \varphi_{t t}+\int_{0}^{+\infty} g(s)\left(a \varphi_{x t}(t-s)\right)_{x} d s=0 \\
\rho_{2} \psi_{t t t}-k_{2} \psi_{x x t}+k_{1}\left(\varphi_{x t}+\psi_{t}\right)=0 \\
\varphi_{t}(0, t)=\psi_{x t}(0, t)=\varphi_{t}(L, t)=\psi_{x t}(L, t)=0
\end{array}\right.
$$

System (7.1) is well posed for initial data $U_{0} \in D(A)$ thanks to Theorem 2.3. Let $E_{2}$ be the second-order energy (the energy of (7.1)) defined by

$$
E_{2}(t)=\frac{1}{2}\left\|U_{t}(t)\right\|_{\mathcal{H}}^{2} .
$$


A simple calculation (as for (4.1) and (4.2)) implies, in case (1.11), that

$$
E_{2}^{\prime}(t) \leq \frac{1}{2} g^{\prime} \circ \varphi_{x t}-\frac{1}{2} \inf _{[0, L]}(b-|d|) \int_{0}^{L} \varphi_{t t}^{2} d x
$$

so $E_{2}$ is non-increasing (according to (1.11)). Let $\tau_{0}=1$ in (5.4). Thus, similarly to (5.5) (with the same choices of $\epsilon_{0}$ and $N$ ), we deduce from (4.38) that

$$
F^{\prime}(t) \leq-\beta_{1} G_{0}(E(t))+\left(\frac{\rho_{1} k_{2}}{k_{1}}-\rho_{2}\right) \frac{G_{0}(E(t))}{E(t)} \int_{0}^{L} \varphi_{x t} \psi_{t} d x .
$$

Now, as in [25], we use some ideas of [17].

Lemma 7.1 For any $\epsilon>0$, we have

$$
\begin{aligned}
& \left(\frac{\rho_{1} k_{2}}{k_{1}}-\rho_{2}\right) \int_{S}^{T} \frac{G_{0}(E(t))}{E(t)} \int_{0}^{L} \varphi_{x t} \psi_{t} d x d t \leq \epsilon \int_{S}^{T} G_{0}(E(t)) d t \\
& +c_{\epsilon} \frac{G_{0}(E(0))}{E(0)}\left(E(S)+E_{2}(S)\right), \quad \forall T \geq S \geq 0 .
\end{aligned}
$$

Proof By integration with respect to $t$, we get

$$
\begin{aligned}
& \left(\frac{\rho_{1} k_{2}}{k_{1}}-\rho_{2}\right) \int_{S}^{T} \frac{G_{0}(E(t))}{E(t)} \int_{0}^{L} \varphi_{x t} \psi_{t} d x d t=\left(\frac{\rho_{1} k_{2}}{k_{1}}-\rho_{2}\right)\left[\frac{G_{0}(E(t))}{E(t)} \int_{0}^{L} \varphi_{x t} \psi d x\right]_{S}^{T} \\
& -\left(\frac{\rho_{1} k_{2}}{k_{1}}-\rho_{2}\right) \int_{S}^{T}\left(\frac{G_{0}(E(t))}{E(t)}\right)^{\prime} \int_{0}^{L} \varphi_{x t} \psi d x d t \\
& -\left(\frac{\rho_{1} k_{2}}{k_{1}}-\rho_{2}\right) \int_{S}^{T} \frac{G_{0}(E(t))}{E(t)} \int_{0}^{L} \varphi_{x t t} \psi d x d t
\end{aligned}
$$

Moreover, applying Poincaré's inequality (2.5), for $\psi$, and using the definition of $E$ and $E_{2}$ and their non-increasingness, we find

$$
\begin{aligned}
& \left|\left(\frac{\rho_{1} k_{2}}{k_{1}}-\rho_{2}\right) \int_{0}^{L} \varphi_{x t} \psi d x\right| \leq c\left(E(t)+E_{2}(t)\right) \\
& \quad \leq c\left(E(S)+E_{2}(S)\right), \quad \forall 0 \leq S \leq t .
\end{aligned}
$$

Thus, by integrating by parts the last integral in (7.6) with respect to $x$ and noting that $\frac{G_{0}(E)}{E}$ is non-increasing, we have

$$
\begin{aligned}
& \left(\frac{\rho_{1} k_{2}}{k_{1}}-\rho_{2}\right) \int_{S}^{T} \frac{G_{0}(E(t))}{E(t)} \int_{0}^{L} \varphi_{x t} \psi_{t} d x d t \\
& \leq c \frac{G_{0}(E(0))}{E(0)}\left(E(S)+E_{2}(S)\right)+c \int_{S}^{T} \frac{G_{0}(E(t))}{E(t)} \int_{0}^{L}\left|\varphi_{t t}\right|\left|\psi_{x}\right| d x d t, \forall T \geq S \geq 0 .
\end{aligned}
$$

On the other hand, according to (1.11) and (7.3) (notice also that $g$ is non-incresing), we have

$$
\int_{0}^{L} \varphi_{t t}^{2} d x \leq \frac{-2}{\inf _{[0, L]}(b-|d|)} E_{2}^{\prime}(t)
$$

\section{Springer}


Then, using (2.14) and Young's inequality (3.1), we estimate the last integral in (7.7) as follows:

$$
\begin{aligned}
& c \int_{S}^{T} \frac{G_{0}(E(t))}{E(t)} \int_{0}^{L}\left|\varphi_{t t}\right|\left|\psi_{x}\right| d x d t \leq \frac{\epsilon \hat{k}}{2} \int_{S}^{T} \frac{G_{0}(E(t))}{E(t)} \psi_{x}^{2} d x d t-c_{\epsilon} \frac{G_{0}(E(0))}{E(0)} \int_{S}^{T} E_{2}^{\prime}(t) d t \\
& \quad \leq \epsilon \int_{S}^{T} G_{0}(E(t)) d t+c_{\epsilon} \frac{G_{0}(E(0))}{E(0)} E_{2}(S), \quad \forall T \geq S \geq 0 .
\end{aligned}
$$

Inserting this inequality into (7.7), we get (7.5).

Now, exploiting (7.4) and (7.5) and choosing $\epsilon \in] 0, \beta_{1}\left[\right.$, we get, for $\beta_{3}=\beta_{1}-\epsilon$,

$$
\int_{S}^{T} F^{\prime}(t) d t \leq-\beta_{3} \int_{S}^{T} G_{0}(E(t)) d t+c \frac{G_{0}(E(0))}{E(0)}\left(E(S)+E_{2}(S)\right), \quad \forall T \geq S \geq 0 .
$$

By combining (7.8) and the property $F \sim E$, we deduce that, for some positive constant $\beta_{4}$,

$$
\int_{S}^{T} G_{0}(E(t)) d t \leq \beta_{4}\left(1+\frac{G_{0}(E(0))}{E(0)}\right)\left(E(S)+E_{2}(S)\right), \quad \forall T \geq S \geq 0 .
$$

Choosing $S=0$ in (7.9) and using the fact that $G_{0}(E)$ is non-increasing, we get

$$
G_{0}(E(T)) T \leq \int_{0}^{T} G_{0}(E(t)) d t \leq \beta_{4}\left(1+\frac{G_{0}(E(0))}{E(0)}\right)\left(E(0)+E_{2}(0)\right), \quad \forall T \geq 0,
$$

which gives (2.41), for $n=1$, with $c_{1}=\beta_{4}\left(1+\frac{G_{0}(E(0))}{E(0)}\right)\left(E(0)+E_{2}(0)\right)$, since $G_{0}^{-1}$ is increasing.

By induction on $n$, suppose that (2.41) holds and let $U_{0} \in D\left(A^{n+1}\right)$ such that $a \equiv 0$ or (2.8) holds or (2.40) holds, for $n+1$ instead of $n$. We have $U_{t}(0) \in D\left(A^{n}\right)$ (thanks to Theorem 2.3) and $U_{t}$ satisfies the first two equations and the boundary conditions of (1.1). On the other hand, if $a \neq 0$ and (2.8) does not hold, then $U_{t}(0)$ satisfies (2.40) (because $U_{0}$ satisfies (2.40), for $n+1$ ). Then the energy $E_{2}$ of (7.1) (defined in (7.2)) also satisfies, for some positive constant $\tilde{c}_{n}$,

$$
E_{2}(t) \leq G_{n}\left(\frac{\tilde{c}_{n}}{t}\right), \quad \forall t>0
$$

Now, choosing $S=\frac{T}{2}$ in (7.9), combining with (2.41) and (7.10), and using the fact that $G_{0}(E)$ is non-increasing, we deduce that

$G_{0}(E(T)) T \leq 2 \int_{\frac{T}{2}}^{T} G_{0}(E(t)) d t \leq 2 \beta_{4}\left(1+\frac{G_{0}(E(0))}{E(0)}\right)\left(G_{n}\left(\frac{2 c_{n}}{T}\right)+G_{n}\left(\frac{2 \tilde{c}_{n}}{T}\right)\right)$,

this implies that, for $c_{n+1}=\max \left\{4 \beta_{4}\left(1+\frac{G_{0}(E(0))}{E(0)}\right), 2 c_{n}, 2 \tilde{c}_{n}\right\}$ (notice that $G_{n}$ is increasing),

$$
E(T) \leq G_{0}^{-1}\left(\frac{c_{n+1}}{T} G_{n}\left(\frac{c_{n+1}}{T}\right)\right)=G_{1}\left(\frac{c_{n+1}}{T} G_{n}\left(\frac{c_{n+1}}{T}\right)\right)=G_{n+1}\left(\frac{c_{n+1}}{T}\right) .
$$

This proves (2.41), for $n+1$. The proof of Theorem 2.7 is completed. 


\section{General comments and issues}

We give in this last section some general comments and issues.

Remark 8.1 When (1.2) does not hold and (1.12) holds, proving the stability of (1.1) seems a delicate question (even under smallness condition on $\|d\|_{\infty}$ ). In this case, there is a double difficulty: the presence of the last term in (4.38) which can not be absorbed by $E$ itself and the fact that (1.1) and (7.1) are not neccessarily dissipative with respect to $E$ and $E_{2}$, respectively (see (4.2) and (7.2)).

Remark 8.2 The regularity $g \in C^{1}\left(\mathbb{R}_{+}\right)$can be weaken by assuming that $g$ is differentiable almost everywhere on $\mathbb{R}_{+}$. On the other hand, our condition (2.9) implies that the set

$$
\left\{s \in \mathbb{R}_{+}, g(s)>0 \text { and } g^{\prime}(0)=0\right\}
$$

is empty. Using the arguments of [64-68], our stability results can be extended to the case of convolution kernels $g$ having flat zones up to a certain extent; that is, the set (8.1) is not negligeable but small enough in some sense.

Remark 8.3 It is interesting to determine the biggest value of $d_{0}$ in (2.39) which guarantees the exponential stability (2.37) of (1.1) when (1.2) and (1.12) hold. On the other hand, is the system (1.1) instable when (1.2) and (1.12) hold, but $\|d\|_{\infty}$ is not small enough?

Remark 8.4 Another interesting question concerns the stability of (1.1) with an additional discrete time delay $\tilde{d} \psi_{t}(t-\tilde{\tau})$ considered on the second equation, where $\tilde{\tau}$ is a positive constant and $\tilde{d}:[0, L] \rightarrow \mathbb{R}$ is a given function.

Remark 8.5 The arguments applied in [20] to get the stability of (1.10) can be adapted to (1.1) and a general stability estimate can be proved when (1.2), (1.12) and (2.9) hold (so $g$ can converge to zero at infinity less faster than exponentially). The arguments of [20] are based on an approach introduced and developped in [64-68]. This approach allowed us to deal with some arbitrary decaying kernels $g$ without assuming explicit conditions on their derivatives $g^{\prime}$ and to avoid passing by $E^{\prime}$ in objective to overcome subsequently the difficulties generated by the non-dissipativeness character of (1.10). On the other hand, the arguments of [20] can be used to obtain the stability of (1.1) in case where the discrete time delay $d \varphi_{t}(t-\tau)$ is replaced by a distributed one

$$
\int_{0}^{+\infty} f(s) \varphi_{t}(t-s) d s,
$$

for some given function $f: \mathbb{R}_{+} \rightarrow \mathbb{R}$. Moreover, the results of the present paper remain true if we replace the linear damping $b \varphi_{t}$ by a non-linear one $b h\left(\varphi_{t}\right)$, for some given function $h: \mathbb{R} \rightarrow \mathbb{R}$. Finally, some other Timoshenko-type systems with controls and time delays on the displacement can be considered (see [25] concerning the case where no delay is considered). To keep away this paper of being too long, we do not discuss these situations.

Remark 8.6 When $\inf _{[0, L]} a>0$ and $\|d\|_{\infty}$ is small enough, the stability estimates (2.34) and (2.41) hold true also in case

$$
\inf _{[0, L]}(b-|d|)=0 .
$$

More precisely, we have the following:

\section{를 Springer}


Theorem 8.7 Assume that $(H 1)-(H 3)$ and (8.2) are satisfied and $\inf _{[0, L]} a>0$. Let

$$
\xi=\left\{\begin{array}{ll}
\tau b & \text { if } d \neq 0, \\
0 & \text { if } d \equiv 0
\end{array} \text { and } \quad \xi_{0}=0\right.
$$

instead of (2.25) and (2.28). Then the well-posedness result of Theorem 2.3 holds true. Moreover, there exists a positive constant $d_{0}$ independent of $d$ such that, if

$$
\|d\|_{\infty}<d_{0}
$$

then

1. Case (1.2) holds: for any $U_{0} \in \mathcal{H}$ such that (2.8) or (2.33) holds, E satisfies (2.34).

2. Case (1.2) does not hold: for any $n \in \mathbb{N}^{*}$ and $U_{0} \in D\left(A^{n}\right)$ such that (2.8) or (2.40) holds, E satisfies (2.41).

Proof First, according to (8.2) and (8.3), (2.27) and (3.2) imply that $B \equiv 0$ and (3.3), respectively. The rest of the proof of Theorem 2.3 is identical to the one given in Sect. 3 .

Second, under the choice (8.3), (4.3) and (8.2) imply that

$$
-g^{\prime} \circ \varphi_{x} \leq-2 E^{\prime}(t)
$$

and

$$
E^{\prime}(t) \leq-\frac{1}{2} \int_{0}^{L} b \varphi_{t}^{2} d x+\frac{\|d\|_{\infty}}{2} \int_{0}^{L} \varphi_{t}^{2} d x
$$

Similarly to (8.5), we have also

$$
-g^{\prime} \circ \varphi_{x t} \leq-2 E_{2}^{\prime}(t)
$$

Because $\xi \leq \tau b$, then

$$
\tilde{c}=c\|d\|_{\infty} \text { and } \tilde{\xi}=\|b\|_{\infty} b
$$

instead of (4.34) and (4.35). Consequently, using (8.6), we have

$$
N E^{\prime}(t)+c \int_{0}^{L} \tilde{\xi} \varphi_{t}^{2} d x \leq \int_{0}^{L}\left(c \tilde{\xi}-\frac{N}{2} b\right) \varphi_{t}^{2} d x+\frac{N\|d\|_{\infty}}{\rho_{1}} E_{0}(t) .
$$

Therefore, inserting (8.5) and (8.9) into (4.38), we get

$$
\begin{aligned}
\frac{G_{0}(E(t))}{E(t)} I_{6}^{\prime}(t) \leq & -\frac{G_{0}(E(t))}{E(t)}\left(\left(c-\frac{N\|d\|_{\infty}}{\rho_{1}}-\tilde{\epsilon}_{0}\right) E_{0}(t)+\left(\tilde{c}-\tilde{\epsilon}_{0}\right) E_{1}(t)\right) \\
& -c\left(1+G^{\prime}\left(\epsilon_{0} E(t)\right)\right) E^{\prime}(t)+\frac{G_{0}(E(t))}{E(t)} \int_{0}^{L}\left(c \tilde{\xi}-\frac{N}{2} b\right) \varphi_{t}^{2} d x \\
& +\left(\frac{\rho_{1} k_{2}}{k_{1}}-\rho_{2}\right) \frac{G_{0}(E(t))}{E(t)} \int_{0}^{L} \varphi_{x t} \psi_{t} d x
\end{aligned}
$$

Choosing $N \geq 0$ such that

$$
c \tilde{\xi}-\frac{N}{2} b \leq 0 \text { and } N>\beta ;
$$


so $N$ can be taken as in (6.3), therefore $I_{6} \sim E$ (due to (4.40)) and, for some positive constant $\beta_{5}$ which does not depend neither on $b$ nor on $d$ (notice that $G^{\prime}\left(\epsilon_{0} E\right)$ is non-increasing),

$$
\begin{aligned}
\frac{G_{0}(E(t))}{E(t)} I_{6}^{\prime}(t) \leq & -\frac{G_{0}(E(t))}{E(t)}\left(\left(c-\beta_{5}\left(1+\|b\|_{\infty}\right)\|d\|_{\infty}-\tilde{\epsilon}_{0}\right) E_{0}(t)+\left(\tilde{c}-\tilde{\epsilon}_{0}\right) E_{1}(t)\right) \\
& -c E^{\prime}(t)+\left(\frac{\rho_{1} k_{2}}{k_{1}}-\rho_{2}\right) \frac{G_{0}(E(t))}{E(t)} \int_{0}^{L} \varphi_{x t} \psi_{t} d x .
\end{aligned}
$$

Next, exploiting (8.4), for $d_{0}=\frac{c}{\beta_{5}\left(1+\|b\|_{\infty}\right)}$, and choosing $\epsilon>0$ such that

$$
c-\beta_{5}\left(1+\|b\|_{\infty}\right)\|d\|_{\infty}-\tilde{\epsilon}_{0}>0 \text { and } \tilde{c}-\tilde{\epsilon}_{0}>0,
$$

we deduce from (8.11) that, for some positive constant $\beta_{6}$,

$$
\frac{G_{0}(E(t))}{E(t)} I_{6}^{\prime}(t)+c E^{\prime}(t) \leq-\beta_{6} G_{0}(E(t))+\left(\frac{\rho_{1} k_{2}}{k_{1}}-\rho_{2}\right) \frac{G_{0}(E(t))}{E(t)} \int_{0}^{L} \varphi_{x t} \psi_{t} d x .
$$

If (1.2) holds, then (8.12) coincides with (5.3) and the proof of (2.34) can be finished as in Sect. 5 .

If (1.2) does not hold, we consider the functional $F$ defined in (5.4) with $\tau_{0}=1$, and then (8.12) becomes identical to (7.4). Consequently, the proof of (2.41) can be ended as in Sect. 7.

\section{References}

1. Alabau-Boussouira, F.: On convexity and weighted integral inequalities for energy decay rates of nonlinear dissipative hyperbolic systems. Appl. Math. Optim. 51, 61-105 (2005)

2. Alabau-Bousosuira, F.: Asymptotic behavior for Timoshenko beams subject to a single nonlinear feedback control. Nonlinear Differ. Equ. Appl. 14, 643-669 (2007)

3. Alabau-Boussouira, F., Cannarsa, P., Komornik, V.: Indirect internal stabilization of weakly coupled evolution equations. J. Evol. Equ. 2, 127-150 (2002)

4. Almeida Júnior, D.S., Santos, M.L., Muñoz Rivera, J.E.: Stability to weakly dissipative Timoshenko systems. Math. Methods Appl. Sci. 36, 1965-1976 (2013)

5. Almeida Júnior, D.S., Santos, M.L., Muñoz Rivera, J.E.: Stability to 1-D thermoelastic Timoshenko beam acting on shear force. Z. Angew. Math. Phys. 65, 1233-1249 (2014)

6. Ammari, K., Nicaise, S., Pignotti, C.: Feedback boundary stabilization of wave equations with interior delay. Syst. Control Lett. 59, 623-628 (2010)

7. Ammar-Khodja, F., Benabdallah, A., Muñoz Rivera, J.E., Racke, R.: Energy decay for Timoshenko systems of memory type. J. Differ. Equ. 194, 82-115 (2003)

8. Apalara, T.A., Messaoudi, S.A., Mustafa, M.I.: Energy decay in Thermoelasticity type III with viscoelastic damping and delay term. Electron. J. Differ. Equ. 128, 1-15 (2012)

9. Benaissa, A., Benaissa, A.K., Messaoudi, S.A.: Global existence and energy decay of solutions for the wave equation with a time varying delay term in the weakly nonlinear internal feedbacks. J. Math. Phys. 53, $123514(2012)$

10. Cavalcanti, M.M., Oquendo, H.P.: Frictional versus viscoelastic damping in a semilinear wave equation. SIAM J. Control Optim. 42, 1310-1324 (2003)

11. Dafermos, C.M.: Asymptotic stability in viscoelasticity. Arch. Ration. Mech. Anal. 37, 297-308 (1970)

12. Datko, R., Lagnese, J., Polis, M.P.: An example on the effect of time delays in boundary feedback stabilization of wave equations. SIAM J. Control Optim. 1, 152-156 (1986)

13. Datko, R.: Two questions concerning the boundary control of certain elastic systems. J. Differ. Equ. 1, 27-44 (1991)

14. Fernández Sare, H.D., Muñoz Rivera, J.E.: Stability of Timoshenko systems with past history. J. Math. Anal. Appl. 339, 482-502 (2008)

15. Fernández Sare, H.D., Racke, R.: On the stability of damped Timoshenko systems: Cattaneo versus Fourier's law. Arch. Ration. Mech. Anal. 194, 221-251 (2009)

\section{Springer}


16. Guesmia, A.: Asymptotic stability of abstract dissipative systems with infinite memory. J. Math. Anal. Appl. 382, 748-760 (2011)

17. Guesmia, A.: On the stabilization for Timoshenko system with past history and frictional damping controls. Palest. J. Math. 2, 187-214 (2013)

18. Guesmia, A.: Well-posedness and exponential stability of an abstract evolution equation with infinite memory and time delay. IMA J. Math. Control Inf. 30, 507-526 (2013)

19. Guesmia, A.: Asymptotic behavior for coupled abstract evolution equations with one infinite memory. Appl. Anal. 94, 184-217 (2015)

20. Guesmia, A.: Some well-posedness and general stability results in Timoshenko systems with infinite memory and distributed time delay. J. Math. Phys. 55, 1-40 (2014)

21. Guesmia, A., Messaoudi, S.A.: On the control of solutions of a viscoelastic equation. Appl. Math. Comput. 206, 589-597 (2008)

22. Guesmia, A., Messaoudi, S.A.: General energy decay estimates of Timoshenko systems with frictional versus viscoelastic damping. Math. Methods Appl. Sci. 32, 2102-2122 (2009)

23. Guesmia, A., Messaoudi, S.A.: On the stabilization of Timoshenko systems with memory and different speeds of wave propagation. Appl. Math. Comput. 219, 9424-9437 (2013)

24. Guesmia, A., Messaoudi, S.A.: A general stability result in a Timoshenko system with infinite memory: a new approach. Math. Methods Appl. Sci. 37, 384-392 (2014)

25. Guesmia, A., Messaoudi, S.A.: Some stability results for Timoshenko systems with cooperative frictional and infinite-memory dampings in the displacement. Acta. Math. Sci. 36, 1-33 (2016)

26. Guesmia, A., Messaoudi, S.A., Soufyane, A.: Stabilization of a linear Timoshenko system with infinite history and applications to the Timoshenko-heat systems. Electron. J. Differ. Equ. 2012, 1-45 (2012)

27. Guesmia, A., Messaoudi, S.A., Wehbe, A.: Uniform decay in mildly damped Timoshenko systems with non-equal wave speed propagation. Dyn. Syst. Appl. 21, 133-146 (2012)

28. Guesmia, A., Tatar, N.E.: Some well-posedness and stability results for abstract hyperbolic equations with infinite memory and distributed time delay. Commun. Pure Appl. Anal. 14, 457-491 (2015)

29. Kafini, M., Messaoudi, S.A., Mustafa, M.I.: Energy decay result in a Timoshenko-type system of thermoelasticity of type III with distributive delay. J. Math. Phys. 54, 101503 (2013)

30. Kafini, M., Messaoudi, S.A., Mustafa, M.I.: Energy decay rates for a Timoshenko-type system of thermoelasticity of type III with constant delay. Appl. Anal. 93, 1201-1216 (2014)

31. Kim, J.U., Renardy, Y.: Boundary control of the Timoshenko beam. SIAM J. Control Optim. 25, 14171429 (1987)

32. Kirane, M., Said-Houari, B., Anwar, M.N.: Stability result for the Timoshenko system with a time-varying delay term in the internal feedbacks. Commun. Pure Appl. Anal. 10, 667-686 (2011)

33. Komornik, V.: Exact Controllability and Stabilization. The Multiplier Method. Masson-John Wiley, Paris (1994)

34. Lasiecka, I., Messaoudi, S.A., Mustafa, M.I.: Note on intrinsic decay rates for abstract wave equations with memory. J. Math. Phys. 54, 1-18 (2013)

35. Lasiecka, I., Tataru, D.: Uniform boundary stabilization of semilinear wave equations with nonlinear boundary damping. Differ. Integral Equ. 6, 507-533 (1993)

36. Lasiecka, I., Toundykov, D.: Regularity of higher energies of wave equation with nonlinear localized damping and source terms. Nonlinear Anal. TMA 69, 898-910 (2008)

37. Liu, W.J., Zuazua, E.: Decay rates for dissipative wave equations. Ricerche di Matematica XLVIII, 61-75 (1999)

38. Messaoudi, S.A., Apalara, T.A.: Asymptotic stability of thermoelasticity type III with delay term and infinite memory. IMA J. Math. Control Inf. 32, 75-95 (2015)

39. Messaoudi, S.A., Michael, P., Said-Houari, B.: Nonlinear Damped Timoshenko systems with second: global existence and exponential stability. Math. Methods Appl. Sci. 32, 505-534 (2009)

40. Messaoudi, S.A., Mustafa, M.I.: On the internal and boundary stabilization of Timoshenko beams. Nonlinear Differ. Equ. Appl. 15, 655-671 (2008)

41. Messaoudi, S.A., Mustafa, M.I.: On the stabilization of the Timoshenko system by a weak nonlinear dissipation. Math. Methods Appl. Sci. 32, 454-469 (2009)

42. Messaoudi, S.A., Mustafa, M.I.: A stability result in a memory-type Timoshenko system. Dyn. Syst. Appl. 18, 457-468 (2009)

43. Messaoudi, S.A., Said-Houari, B.: Uniform decay in a Timoshenko-type system with past history. J. Math. Anal. Appl. 360, 459-475 (2009)

44. Muñoz Rivera, J.E., Racke, R.: Mildly dissipative nonlinear Timoshenko systems-global existence and exponential stability. J. Math. Anal. Appl. 276, 248-278 (2002)

45. Muñoz Rivera, J.E., Racke, R.: Global stability for damped Timoshenko systems. Discrete Contin. Dyn. Syst. 9, 1625-1639 (2003) 
46. Muñoz Rivera, J.E., Racke, R.: Timoshenko systems with indefinite damping. J. Math. Anal. Appl. 341, $1068-1083(2008)$

47. Mustafa, M.I., Messaoudi, S.A.: General energy decay rates for a weakly damped Timoshenko system. Dyn. Control Syst. 16, 211-226 (2010)

48. Nicaise, S., Pignotti, C.: Stability and instability results of the wave equation with a delay term in the boundary or internal feedbacks. SIAM J. Control Optim. 5, 1561-1585 (2006)

49. Nicaise, S., Pignotti, C.: Stabilization of the wave equation with boundary or internal distributed delay. Differ. Integral Equ. 9-10, 935-958 (2008)

50. Nicaise, S., Pignotti, C.: Interior feedback stabilization of wave equations with time dependent delay. Electron. J. Differ. Equ. 41, 1-20 (2011)

51. Nicaise, S., Pignotti, C., Valein, J.: Exponential stability of the wave equation with boundary time-varying delay. Discrete Contin. Dyn. Syst. Ser. S 3, 693-722 (2011)

52. Nicaise, S., Valein, J., Fridman, E.: Stability of the heat and of the wave equations with boundary timevarying delays. Discrete Contin. Dyn. Syst. Ser. S 2, 559-581 (2009)

53. Pazy, A.: Semigroups of Linear Operators and Applications to Partial Differential Equations. Springer, New York (1983)

54. Racke, R., Said-Houari, B.: Global existence and decay property of the Timoshenko system in thermoelasticity with second sound. Nonlinear Anal. 75, 4957-4973 (2012)

55. Racke, R., Said-Houari, B.: Decay rates and global existence for semilinear dissipative Timoshenko systems. Q. Appl. Math. 71, 229-266 (2013)

56. Raposo, C.A., Ferreira, J., Santos, M.L., Castro, N.N.O.: Exponential stability for the Timoshenko system with two week dampings. Appl. Math. Lett. 18, 535-541 (2005)

57. Said-Houari, B.: A stability result for a Timoshenko system with past history and a delay term in the internal feedback. Dyn. Syst. Appl. 20, 327-354 (2011)

58. Said-Houari, B., Kasimov, A.: Decay property of Timoshenko system in thermoelasticity. Math. Methods Appl. Sci. 35, 314-333 (2012)

59. Said-Houari, B., Kasimov, A.: Damping by heat conduction in the Timoshenko system: Fourier and Cattaneo are the same. J. Differ. Equ. 255, 611-632 (2013)

60. Said-Houari, B., Laskri, Y.: A stability result of a Timoshenko system with a delay term in the internal feedback. Appl. Math. Comput. 217, 2857-2869 (2010)

61. Said-Houari, B., Soufyane, A.: Stability result of the Timoshenko system with delay and boundary feedback. IMA J. Math. Control Inf. 29, 383-398 (2012)

62. Santos, M.L., Almeida Júnior, D.S., Muñoz Rivera, J.E.: The stability number of the Timoshenko system with second sound. J. Differ. Equ. 253, 2715-2733 (2012)

63. Soufyane, A., Wehbe, A.: Uniform stabilization for the Timoshenko beam by a locally distributed damping. Electron. J. Differ. Equ. 29, 1-14 (2003)

64. Tatar, N.E.: Exponential decay for a viscoelastic problem with a singular kernel. Z. Angew. Math. Phys. 60, 640-650 (2009)

65. Tatar, N.E.: On a large class of kernels yielding exponential stability in viscoelasticity. Appl. Math. Comput. 215, 2298-2306 (2009)

66. Tatar, N.E.: How far can relaxation functions be increasing in viscoelastic problems? Appl. Math. Lett. 22, 336-340 (2009)

67. Tatar, N.E.: A new class of kernels leading to an arbitrary decay in viscoelasticity. Mediterr. J. Math. 6, 139-150 (2010)

68. Tatar, N.E.: On a perturbed kernel in viscoelasticity. Appl. Math. Lett. 24, 766-770 (2011)

69. Timoshenko, S.: On the correction for shear of the differential equation for transverse vibrations of prismaticbars. Philis. Mag. 41, 744-746 (1921) 
Journal: 13370

Article: 514

\section{Springer}

the language of science

\section{Author Query Form}

\section{Please ensure you fill out your response to the queries raised below}

and return this form along with your corrections

\section{Dear Author}

During the process of typesetting your article, the following queries have arisen. Please check your typeset proof carefully against the queries listed below and mark the necessary changes either directly on the proof/online grid or in the 'Author's response' area provided below

\begin{tabular}{|c|l|c|}
\hline Query & \multicolumn{1}{|c|}{ Details required } & Author's response \\
\hline 1. & $\begin{array}{l}\text { Kindly check the abbreviated journal title } \\
\text { for the references }[45,47] .\end{array}$ & \\
\hline
\end{tabular}

